\title{
NASH EQUILIBRIUM DESIGN AND PRICE-BASED COORDINATION IN HIERARCHICAL SYSTEMS
}

\author{
MiCHAŁ P. KARPOWICZ \\ Research and Academic Computer Network (NASK) \\ ul. Wąwozowa 18, 02-796 Warsaw, Poland \\ Institute of Control and Computation Engineering \\ Warsaw University of Technology, Nowowiejska 15/19, 00-665 Warsaw, Poland \\ e-mail: M.Karpowicz@elka.pw.edu.pl
}

\begin{abstract}
This paper deals with the problem of designing Nash equilibrium points in noncooperative games in which agents anticipate values of Lagrange multipliers coordinating their payoff functions. The addressed model of agents' interactions, referred to as the price-anticipation game, is studied within the framework of coordination and mechanism design theory for hierarchical systems. Sufficient conditions are formulated for Nash implementation of a regular and isolated solution to a coordination problem. An equilibrium design procedure is proposed and applied as an analytic tool in a study of mechanism design games. In the setting considered the well-known fact is demonstrated that gains from reaching a desired solution to a coordination problem in a Nash equilibrium point need not balance the overall costs of its implementation. However, it is also demonstrated how these costs can be distributed among the agents and related to the particular organization of interactions in the system. Finally, application of the developed framework in the field of Internet traffic engineering is presented.
\end{abstract}

Keywords: Nash equilibrium, coordination in hierarchical systems, decentralized control, asymmetric information.

\section{Introduction}

A game is formally defined as a mathematical model of interaction (competition or cooperation) of active agents (autonomous, intelligent and rational in a specified sense) making interrelated choices under imperfect information. The solution concept of a game is any prediction of decisions that may be taken by the agents. The fundamental one is given by the Nash equilibrium (Nash, 1950; 1951). Suppose that there are $n$ agents minimizing payoff (or cost) functions $J_{i}, i=1, \ldots, n$, where each $J_{i}$ depends on the individual decision $x_{i} \in$ $X_{i}$ of agent $i$ and decisions of the others $\mathbf{x}_{-i}=$ $\left(x_{1}, \ldots, x_{i-1}, x_{i+1}, \ldots, x_{n}\right) \in X_{1} \times \cdots \times X_{i-1} \times$ $X_{i+1} \times \cdots \times X_{n}$. The Nash equilibrium is a point $\mathrm{x}^{*}=$ $\left(x_{1}^{*}, \ldots, x_{n}^{*}\right)$ at which the following system of inequalities is satisfied:

$$
J_{i}\left(x_{i}^{*}, \mathbf{x}_{-i}^{*}\right) \leq J_{i}\left(s_{i}, \mathbf{x}_{-i}^{*}\right), \quad s_{i} \in X_{i}, i=1, \ldots, n .
$$

According to the above definition there is no profitable deviation from $x_{i}^{*}, i=1, \ldots, n$, individually available for any agent in a Nash equilibrium point $\mathrm{x}^{*}$.
For an extensive discussion of the main concepts and applications of game theory, see the works of Myerson (1991), Fudenberg and Tirole (1991), Basar and Olsder (1999), as well as Mas-Colell et al. (1995). This paper deals with the problem of designing Nash equilibrium points in games that may arise in hierarchical systems in which coordination mechanisms are applied that exploit dual variables, referred to as prices, as coordination signals.

Consider a decentralized system, sketched in Fig. 1 , of $n$ interacting plants controlled by $n$ active agents. The system is managed by the coordinator, also referred to as the mechanism designer or the system regulator, capable of defining the rules of plants' interactions. The coordinator is faced with the problem of designing a mechanism $\mathbf{m}$ that leads the interacting plants, controlled by the agents, to a solution of a coordination problem, call it $(\mathrm{P})$. The agents are competing with each other to reach their private goals related to optimization of functions $f_{0 i}, i=1, \ldots, n$.

The coordination problem, usually a multiobjective 
one, represents the coordinator's preferences defined with respect to performance of the system. The performance index is assumed to be given by a scalarizing function $f_{0}$ aggregating individual preferences $f_{0 i}, i=1, \ldots, n$, of the agents. As far as the engineering practice is considered, there are two performance measures that are usually applied, namely, expressing the egalitarian or utilitarian fairness concept (see the works of Wierzbicki et al. (2001), Pióro and Medhi (2004), Ogryczak et al. (2005; 2008) for networking and decision support applications, and those of Sen $(1969 ; 1970 ; 1977)$ for general characterization of the fairness concept). In this paper, we limit our discussion to the coordination goal defined by the utilitarian aggregation (with equal weights), $f_{0}=\sum_{i=1}^{n} f_{0 i}$. Furthermore, due to a local scope of our analysis, we do not infer Pareto-optimality of the studied solutions.

The agents interacting in the system are assumed to be active, which means that they are capable of making autonomous decisions (however, subject to the constraints defined by mechanism $\mathbf{m}$ ). As a consequence, it is necessary to make some, rather strong, assumptions regarding their decision making processes. Our focus is, therefore, limited to the environments in which the agents try to reach a Nash equilibrium point.

The central assumption in our investigations, naturally satisfied in many real-life systems, is that the agents' preferences, $f_{0 i}, i=1, \ldots, n$, are not commonly known (see Rotschild and Stiglitz, 1976; Grossman and Stiglitz, 1980; Stiglitz, 2000). Precisely, the preference indicator $f_{0 i}$ is required to be privately known only to the $i$-th agent. Let us briefly point out implications that this assumption may have for coordinability of the system, i.e., reachability of the coordination goal.

As can be noticed, in the addressed asymmetric information environment the coordinator must rest his/her decisions on information that the agents decide to reveal. However, the agents may find it profitable to take advantage of the monopoly they have on the knowledge regarding their individual preferences. In order to accomplish this goal, the agents, referring to the idea underlying the preference revelation principle (Green and Laffont, 1979; Myerson, 1981; Mas-Colell et al., 1995), may dishonestly communicate to the coordinator a suitably modified profile of preferences $\tilde{f}_{0 i} \neq f_{0 i}, i=$ $1, \ldots, n$. Equivalently, they may try to apply the controls $\tilde{x}_{i}, i=1, \ldots, n$, that optimize $\tilde{f}_{0 i}$ instead of $f_{0 i}$. Suppose that there exists an equilibrium point that is reachable under such control strategies. Clearly, its efficiency will then be determined by the aggregation of the fictitious preferences, $f_{0}=\sum_{i=1}^{n} \tilde{f}_{0 i}$, observed by the coordinator. In other words, the actual performance of the system, described by $f_{0}=\sum_{i=1}^{n} f_{0 i}$, will not be optimized. At the same time, though, as illustrated later in the paper, in the equilibrium some of the agents may improve

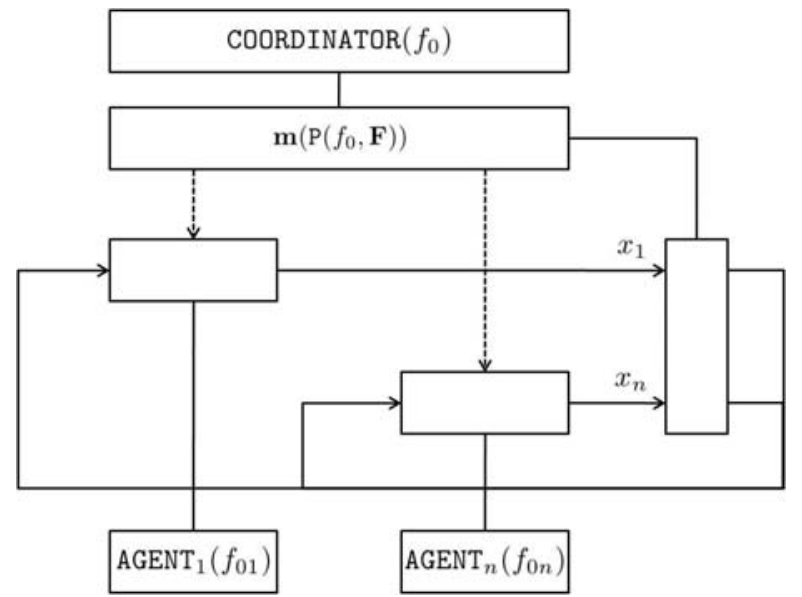

Fig. 1. Structure of a decentralized coordination system.

their payoffs above those attainable if true preferences were revealed. Consequently, in order to reach the coordination goal, mechanism $\mathbf{m}$, defining the rules of the agents' interactions, should create sufficient incentives for the agents to apply the controls that indeed optimize $f_{0 i}, i=1, \ldots, n$. For a classic discussion of the theory of incentives, see the works of Arrow and Hurwicz (1977), Green and Laffont (1979), Mas-Colell et al. (1995), as well as Laffont and Martimort (2002).

The mechanisms under our consideration exploit the Lagrange multipliers, associated with the interaction balancing constraints in the system, as a feedback to the agents. Namely, we study the properties of mechanisms specifying the coordination procedures, also referred to as auctions, in which in the course of an iterative process the coordination signals, interpreted as prices, lead the interacting agents to outcomes optimizing their preferences. In this paper, however, it is not the dynamics of the process that are under our investigation but the static properties of its equilibrium points.

More formally, we search for the sufficient conditions that must be satisfied by a local solution to the coordination problem:

$$
\text { (P) } \begin{cases}\text { minimize } & f_{0}(\mathbf{x}, \mathbf{y}) \text { over }(\mathbf{x}, \mathbf{y}) \in \mathbb{R}^{n} \times \mathbb{R}^{m}, \\ \text { subject to } & f_{j}(\mathbf{x}, \mathbf{y})=0, j=1, \ldots, m,\end{cases}
$$

to be reachable in a pure-strategy Nash equilibrium point of the noncooperative game in which active agents anticipate values of the Lagrange multipliers associated with the constraints $f_{j}, j=1, \ldots, m$, at the solution. We refer to this model of interaction as the price-anticipation game.

A motivation for our studies comes from the following observation. Suppose that $(\overline{\mathbf{x}}, \overline{\mathbf{y}}) \in \mathbb{R}^{n} \times \mathbb{R}^{m}$ is a solution to $(\mathrm{P})$ and in a neighborhood $\mathbb{B}_{\epsilon}(\overline{\mathbf{x}}) \times \mathbb{B}_{\epsilon}(\overline{\mathbf{y}})$ of $(\overline{\mathbf{x}}, \overline{\mathbf{y}})$, with $\mathbb{B}_{\epsilon}(\overline{\mathbf{x}})=\{\mathbf{x}:\|\overline{\mathbf{x}}-\mathbf{x}\| \leq \epsilon, \epsilon>0\}$, functions 
$f_{j}: \mathbb{R}^{n} \times \mathbb{R}^{m} \rightarrow \mathbb{R}, j=0, \ldots, m$, are continuously differentiable with respect to all arguments. Let us also assume that

$$
\operatorname{det} \frac{\partial \mathbf{F}^{T}}{\partial \mathbf{y}}(\overline{\mathbf{x}}, \overline{\mathbf{y}})=\left|\begin{array}{lll}
\frac{\partial f_{1}(\overline{\mathbf{x}}, \overline{\mathbf{y}})}{\partial y_{1}} & \ldots & \frac{\partial f_{m}(\overline{\mathbf{x}}, \overline{\mathbf{y}})}{\partial y_{1}} \\
\ldots \ldots \ldots \ldots \ldots \ldots \ldots & \ldots \ldots \ldots \\
\frac{\partial f_{1}(\overline{\mathbf{x}}, \overline{\mathbf{y}})}{\partial y_{m}} & \ldots & \frac{\partial f_{m}(\overline{\mathbf{x}}, \overline{\mathbf{y}})}{\partial y_{m}}
\end{array}\right| \neq 0,
$$

where $\mathbf{F}=\left(f_{1}, \ldots, f_{m}\right)^{T}$. It follows from the implicit function theorem that for every $\mathbf{x} \in \mathbb{B}_{\epsilon}(\overline{\mathbf{x}})$ we have $\mathbf{y}=\mathbf{Y}(\mathbf{x})$, where $\mathbf{Y}: \mathbb{B}_{\epsilon}(\overline{\mathbf{x}}) \rightarrow \mathbb{B}_{\epsilon}(\overline{\mathbf{y}})$ is continuously differentiable and uniquely defined by the equation $\mathbf{F}(\mathbf{x}, \mathbf{y})=0$. Furthermore, $\overline{\mathbf{y}}=\mathbf{Y}(\overline{\mathbf{x}})$ and $\mathbf{F}(\overline{\mathbf{x}}, \mathbf{Y}(\overline{\mathbf{x}})) \equiv 0$. As a consequence, the problem (P) of constrained optimization over $(\mathbf{x}, \mathbf{y})$ can be locally replaced by that of unconstrained optimization over $\mathrm{x}$. On the other hand, since the regularity condition is satisfied at $(\overline{\mathbf{x}}, \overline{\mathbf{y}})$, the necessary optimality conditions imply the existence of Lagrange multipliers $\overline{\boldsymbol{\mu}}=\left(\bar{\mu}_{1}, \ldots, \bar{\mu}_{m}\right) \in$ $\mathbb{R}^{m}$ such that

$$
\frac{\partial f_{0}}{\partial \mathbf{y}}(\overline{\mathbf{x}}, \overline{\mathbf{y}})+\frac{\partial \mathbf{F}^{T}}{\partial \mathbf{y}}(\overline{\mathbf{x}}, \overline{\mathbf{y}}) \overline{\boldsymbol{\mu}}=0 .
$$

Solution of this equation combined with the local relation $\mathbf{y}=\mathbf{Y}(\mathbf{x})$ yields

$$
\boldsymbol{\mu}=-\left(\frac{\partial \mathbf{F}^{T}}{\partial \mathbf{y}}(\mathbf{x}, \mathbf{Y}(\mathbf{x}))\right)^{-1} \frac{\partial f_{0}}{\partial \mathbf{y}}(\mathbf{x}, \mathbf{Y}(\mathbf{x}))
$$

In other words, in a neighborhood of $(\overline{\mathbf{x}}, \overline{\mathbf{y}})$ multipliers $\bar{\mu}_{j}, j=1, \ldots, m$, can be defined by $\overline{\mathbf{x}}$ only. This fact is at the center of the discussion presented below. It serves as a springboard to a game-theoretic study of outcomes that are reachable when (i) decomposition principle is applied to solve $(\mathrm{P})$ in the decentralized environment described by Fig. 1, and (ii) emerging local plant control subproblems are defined and solved by the agents capable of taking the relation (1) into account.

Finally, we consider systems in which the coordination process can be decentralized and, hence, no central coordination unit is required. As an illustrative example of a mechanism under investigation, consider the TCP protocol commonly used in communication networks (see, e.g., Stallings, 1998). The protocol controls the rate at which a network node sends packets based on locally observed congestion signals. These signals provide feedback information about the utilization of the links in the network and can be interpreted as link capacity prices. It should be noted here that the rate at which a TCP node can send data is determined by a congestion control algorithm specified by the implementation of the TCP protocol (e.g., fast, reno, hstcp, stcp) deployed in the network nodes. However, what implementation is indeed deployed depends on the network users, autonomously managing their network nodes. In other words, there exists an information asymmetry between the network manager (coordinator) and the network users. Recent results in the field of game-theoretic modeling of preferences in hierarchical systems can be found in the work of Kołodziej and Xhafa (2011).

Outline of results. In the following sections we show how to design payoffs $J_{i}\left(x_{i}, \mathbf{x}_{-i}\right), i=1, \ldots, n$, of the price-anticipation game, defined by the condition (1), in order to reach a solution to the coordination problem $(\mathrm{P})$ at a Nash equilibrium point. The condition (1) is assumed to be discovered and exploited by $n$ active agents optimizing their private preference indicators $f_{0 i}\left(x_{i}\right), i=1, \ldots, n$, subject to the imposed rules of interaction.

We begin, in Section 2, by discussing an example of the price-anticipation game induced in the network environment by the rules of a resource allocation mechanism coordinating decisions of active agents by means of dual variables (prices) associated with the resource utilization constraints. It is assumed that the rules of resource allocation and the coordination goal are commonly known. On the other hand, preferences of the agents are assumed to be known only to the agents themselves. In such a setting the agents may share the belief concerning equilibrium conditions that the coordinator tries to reach and, thus, anticipate the influence of their decisions on the coordination signals.

The main result of the paper is Theorem 1 given in Section 3. It specifies sufficient conditions for implementation of a regular local solution to (P) at a Nash equilibrium point of the (noncooperative) price-anticipation game with payoffs parameterized by the price-based coordination instruments (price-based penalty functions or transfer rules). Theorem 1 describes a design procedure that exploits local properties of the feasible solutions to $\mathbf{F}(\mathbf{x}, \mathbf{Y}(\mathbf{x})) \equiv 0$ in order to define a collection of mechanisms that locally coordinate interactions of the agents. Each mechanism in the collection is defined by the interaction balancing rule, $\mathbf{Y}(\mathbf{x})$, and the coordination instruments, $\eta_{i}(\mathbf{x}), i=1, \ldots, n$. By construction these instruments remain invariant with respect to the first-order variations of prices, which preserves compatibility of the coordination goal with the private goals of the interacting price-anticipating agents. In this respect Theorem 1 provides an insight into the relationship between the properties of Lagrange multipliers, the Nash equilibrium of the price-anticipation game and the incentive compatibility (or coordinability) condition.

In Section 4 we demonstrate application of Theorem 1 in a study of the equilibrium design problem defined for a class of quadratic preference indicators. First, we consider a mechanism design game in which 
an agent decides whether to apply the price-anticipating or the price-taking strategy, whereas the coordinator decides whether or not to counterspeculate. This analysis indicates that it may be more desirable for the coordinator to reach a suboptimal solution with the price-anticipating agent than the optimal one imposing additional coordination costs. Second, we show how the costs of counterspeculation can be controlled, at least to some extent, by a proper design of coordination instruments and, in particular, by a choice of the interaction variables in the system. Indeed, by Theorem 1 interactions of the control variables, $\mathbf{Y}(\mathbf{x})$, specify local properties of the equilibrium point.

Finally, in Section 5 we discuss the problem of congestion control in the Internet to illustrate how the presented results refer to potential performance of mechanisms applied in practice. We discuss the design and the properties of the TCP traffic rate control algorithm commonly used in communication networks.

The aim of this paper is to enrich the field of mechanism design theory by an analytic tool supporting the study of price-anticipation games. We also hope that the tools developed in this paper can be of some use in supporting the coordination (mechanism design) decisions. Application fields for which the findings of this paper seem to be useful include distributed hierarchical systems with active agents, especially such as interconnected telecommunication networks and smart power grids. For detailed game-theoretic study of mechanism design problems, see the works of Arrow and Hurwicz (1977), Groves et al. (1987), Green and Laffont (1979), Myerson (1991), Mas-Colell et al. (1995), Krishna (2002), or Milgrom (2004). Other recent results concerning efficiency of the Nash equilibrium in games with price-anticipating agents can be found in the works of Johari and Tsitsiklis (2009), Alpcan and Pavel (2009), Alpcan et al. (2010), Yang and Hajek (2007), or Karpowicz (2010). Finally, the theory of hierarchical systems is presented by Findeisen et al. (1980).

\section{Motivation: Network utility optimization}

Let us describe a price-anticipation game that can be induced in the network environment by the rules of resource allocation mechanisms.

Consider the problem of designing a resource allocation procedure that finds a solution to

$$
(\mathrm{S}) \begin{cases}\operatorname{minimize} & f_{0}(\mathbf{x}, \mathbf{y})=\sum_{j=1}^{m} C_{j}\left(y_{j}\right)-\sum_{i=1}^{n} U_{i}\left(x_{i}\right) \\ \text { subject to } & f_{j}(\mathbf{x}, \mathbf{y})=\sum_{i \in \mathcal{S}(j)} x_{i}-y_{j}=0, \\ \text { over } & (\mathbf{x}, \mathbf{y}) \in \mathbb{R}^{n} \times \mathbb{R}^{m}, j=1, \ldots, m,\end{cases}
$$

in a network of interacting agents. A popular interpretation of this problem relates to congestion-control in communication networks (Kelly, 1997). There are $m$ links in a network shared by $n$ users. Each user $i=1, \ldots, n$ is associated with a connection that can be realized between the selected source-destination pair of network nodes. The connection is established by a collection of paths consisting of network links. User $i$ receives utility $U_{i}\left(x_{i}\right)$ from the transmission rate $x_{i} \geq$ 0 , if each link $j=1, \ldots, m$ on the connection path allocates to the connection a share of its capacity $y_{j} \geq$ 0 . Function $f_{0}$ gives a value of cost that can be attached to allocation $(\mathbf{x}, \mathbf{y})$. It aggregates allocation costs $C_{j}\left(y_{j}\right), j=1, \ldots, m$, and utilities $U_{i}\left(x_{i}\right), i=1, \ldots, n$, into a value underlying judgement about the system performance. The constraints $f_{j}, j=1, \ldots, m$, state that supply $y_{j}$ must satisfy demand $\sum_{i \in S(j)} x_{i}$ revealed by a subset $\mathcal{S}(j) \subseteq\{1, \ldots, n\}$ of consumers. We will refer to this interpretation in the following paragraphs. For convenience, in this section let us also assume strict convexity of the objective in $(\mathrm{S})$.

Suppose that to solve the above problem the coordinator applies a mechanism known as the uniform-price (or Walrasian) auction. As usually, the mechanism is defined by the resource allocation rule and the payment rule. The allocation rule assigns to each network user resources that are available on each link forming the user's connection. The payment rule assigns to each user the cost of the established transmission rate, realized over a subset of network links. These costs are determined by the resource utilization prices that are calculated by the coordinator based on the observed demand for the transmission rate. More formally, the mechanism can be derived from the following decomposition of the Lagrange function:

$$
\begin{aligned}
H(\mathbf{w}, \mathbf{q})= & \sum_{j=1}^{m}\left[C_{j}\left(y_{j}\right)-\mu_{j} y_{j}-\nu_{j} y_{j}\right] \\
& -\sum_{i=1}^{n}\left[U_{i}\left(x_{i}\right)-x_{i} \sum_{j \in \mathcal{D}(i)} \mu_{j}+\lambda_{i} x_{i}\right] \\
= & H_{C}(\mathbf{y}, \boldsymbol{\mu}, \boldsymbol{\nu})-\sum_{i=1}^{n} H_{i}\left(x_{i}, \boldsymbol{\mu}, \lambda_{i}\right)
\end{aligned}
$$

where $\mathbf{w}=(\mathbf{x}, \mathbf{y})$ and $\mathbf{q}=(\boldsymbol{\mu}, \boldsymbol{\nu}, \boldsymbol{\lambda})$.

Suppose now that functions $C_{j}, j=1, \ldots, m$, are commonly known, although the function $U_{i}$ is known only to the $i$-th network user. It can be easily argued that the choice of prices, made by the coordinator, is naturally related to optimization of function $H_{C}$. Indeed, since the agents know what are the true costs of resource allocation, the coordinator would find it difficult to justify any other choice of prices. At the same time, from the viewpoint of the $i$-th network user, the choice of the way in which demand $x_{i}$ is calculated is not 
straightforward, i.e., it does not need to be related to optimization of $H_{i}$. In the asymmetric information setting considered user $i$ may take advantage of the monopoly on information regarding function $U_{i}$, which is required by the coordinator to optimize $H$. For example, in response to the observed prices, communicated by the coordinator, the user may apply a suitably modified function $\tilde{U}_{i} \neq U_{i}$ in order to calculate a profitably reduced level of demand. Since demand reduction may lead to lower prices in the equilibrium, some users may improve their payoffs by applying the above price-anticipating strategy.

In the following paragraphs we will briefly discuss two possible control strategies that can be applied by the users: price-taking and price-anticipating.

Price-taking strategy. A straightforward approach to calculate $x_{i}$ is to optimize $H_{i}$ in response to the vector of fixed prices $\boldsymbol{\mu}$, reflecting the observed state of the network. In such a case user $i$, acting as the price-taker, solves locally the subproblem

$$
\left(\mathbf{S}^{i}(\boldsymbol{\mu})\right)\left\{\begin{array}{l}
\operatorname{maximize} U_{i}\left(x_{i}\right)-\bar{\eta}_{i}\left(\boldsymbol{\mu}, x_{i}\right) \\
\text { over } x_{i} \in X_{i} \subset \mathbb{R}_{+} \\
\text {where } \bar{\eta}_{i}\left(\boldsymbol{\mu}, x_{i}\right) \triangleq x_{i} \sum_{j \in \mathcal{D}(i)} \mu_{j},
\end{array}\right.
$$

coordinated by the values of Lagrange multipliers (prices of network links). If applicability conditions of the price-based coordination are satisfied, then a neighborhood of a solution to $(\mathrm{S})$ may be reached in the course of the following iterative process. Given a vector of fixed prices $\boldsymbol{\mu}(k)$ observed in the network in iteration $k$, each user $i$ solves $\left(\mathbf{S}^{i}(\boldsymbol{\mu}(k))\right)$. Solution $x_{i}(k)$ is then applied in the network that, in response, adjusts prices. For a general study of price-adjustment procedures under price-taking assumption, see, e.g., the works of Negishi (1960), Uzawa (1960), Arrow and Hurwicz (1977), and Findeisen et al. (1980).

Price-anticipating strategy. Although the above pricetaking approach has many advantages, for both the coordinator and the network users, in some cases it may be reasonable for the users to calculate demand in a different way. This can be motivated by the following observation. By assumption, it is commonly known that the network's goal is to reach the steady-state in which prices satisfy the condition (1), i.e., $\mu_{j}=C_{j}^{\prime}\left(y_{j}\right)=p_{j}(\mathbf{x}), j=1, \ldots, m$. A network user occupying more and more of a resource in the network may, therefore, eventually observe his/her nonnegligible impact on the resource price, defined by the above condition. As a consequence, the user may find it reasonable to actively apply the following price- anticipating strategy:

$\left(\mathrm{A}^{i}\right)\left\{\begin{array}{l}\operatorname{maximize} U_{i}\left(x_{i}\right)-\bar{\eta}_{i}\left(\mathbf{p}(\mathbf{x}), x_{i}\right) \\ \text { over } x_{i} \in X_{i} \subset \mathbb{R}_{+}, \\ \text {where } \bar{\eta}_{i}\left(\mathbf{p}(\mathbf{x}), x_{i}\right) \triangleq x_{i} \sum_{j \in \mathcal{D}(i)} p_{j}\left(\sum_{k \in \mathcal{S}(j)} x_{k}\right) .\end{array}\right.$

Notice that the first-order optimality conditions for $\left(\mathrm{A}^{i}\right)$ are now incompatible with the first-order conditions for (S). This brings us to the conclusion that the incentive compatibility (or coordinability) constraint is violated by the rules of the mechanism considered: individual goals of the agents are no longer harmonized with the goal of the coordinator (invisible hand of selfish competition no longer promotes the system efficiency). The desired solution to $(\mathrm{S})$ becomes unreachable in the setting considered.

Let us briefly refer to the question of incentives that a network user may have to anticipate prices and to become involved in the price-anticipation game with other users and the coordinator. Provided that there exists a solution to the game, it may seem that such a strategy could improve individual payoffs or allocations in comparison to those attainable in a solution to $(\mathrm{S})$. Indeed, for the family of strictly convex problems, it was demonstrated by Karpowicz (2010) that there exists a subset of agents that gain by anticipating prices under the rules of the Walrasian market-clearing process. This result is also illustrated in Section 4, in which we show that if the price-anticipating strategy is applied by an agent, then his/her individual payoff may be improved in comparison with the payoff reachable under the price-taking strategy. Other examples and real-life observations of price-anticipating behavior can be found, e.g., in the works of Karpowicz (2011) and Lubacz et al. (2011). See also the contribution by Johari (2004) for a detailed analysis of efficiency loss in market mechanism for resource allocation.

To close this section, let us show that solution to (S) can be reached with price-anticipating users as well. Consider the following modification of $\left(\mathrm{A}^{i}\right)$ :

$\left(\mathrm{A}_{*}^{i}\right)\left\{\begin{array}{l}\operatorname{maximize} U_{i}\left(x_{i}\right)-\bar{\eta}_{i}\left(\mathbf{p}(\mathbf{x}), x_{i}\right) \\ \text { over } x_{i} \in X_{i} \subset \mathbb{R}_{+} \\ \text {where } \bar{\eta}_{i}\left(\mathbf{p}(\mathbf{x}), x_{i}\right) \triangleq \sum_{j \in \mathcal{D}(i)} \int_{0}^{x_{i}} p_{j}\left(s, \mathbf{x}_{-i}\right) \mathrm{d} s .\end{array}\right.$

If the payment rule of the resource allocation mechanism is defined as above, then the first-order optimality conditions for $\left(\mathrm{A}_{*}^{i}\right)$ give rise to outcomes that establish a solution to $(\mathrm{S})$, as desired, even though the price-anticipating strategy is applied by each agent. In other words, the incentive compatibility condition is satisfied under the above rules of price-based 
coordination. This is precisely the observation that motivates our research. In the following section we show how to design price-based coordination instruments that are characterized by the observed property.

\section{Nash equilibrium design}

In this section conditions are given under which a regular solution $\overline{\mathbf{z}}=(\overline{\mathbf{x}}, \overline{\mathbf{y}})$ to

$$
(\mathrm{P}) \begin{cases}\operatorname{minimize} & f_{0}(\mathbf{x}, \mathbf{y}) \text { over }(\mathbf{x}, \mathbf{y}) \in \mathbb{R}^{n} \times \mathbb{R}^{m} \\ \text { subject to } & f_{j}(\mathbf{x}, \mathbf{y})=0, j=1, \ldots, m, \\ & n, m>0, f_{j} \in \mathscr{C}^{2}, j=0, \ldots, m,\end{cases}
$$

can be reached at a Nash equilibrium point of the game defined locally by the system

$$
\left\{\begin{array}{l}
\frac{\partial f_{0}}{\partial \mathbf{y}}(\overline{\mathbf{x}}, \overline{\mathbf{y}})+\frac{\partial \mathbf{F}^{T}}{\partial \mathbf{y}}(\overline{\mathbf{x}}, \overline{\mathbf{y}}) \overline{\boldsymbol{\mu}}=0, \\
\mathbf{F}(\overline{\mathbf{x}}, \overline{\mathbf{y}})=0
\end{array}\right.
$$

determining the Lagrange multipliers associated with the constraints $f_{j}, j=1, \ldots, m$.

The environment of our interest, in which Problem (P) is to be solved, is characterized by the following properties. First, the system coordinator can decompose problem (P) with respect to control (independent) variables $x_{i}, i=1, \ldots, n$, and interaction (dependent) variables $y_{j}, j=1, \ldots, m$. Second, the problem of calculating $x_{i}$ is delegated to a designated agent $i=$ $1, \ldots, n$, whereas the interaction variables $y_{j}, j=$ $1, \ldots, m$, remain managed by the system coordinator. In other words, we consider environments in which control variables can be calculated independently by the agents actively interacting with each other in order to determine the values of interaction variables, establishing solution to $\mathbf{F}(\mathbf{x}, \mathbf{y})=0$, where $\mathbf{y}=\mathbf{Y}(\mathbf{x})$. Finally, the agents actively exploit the first-order optimality conditions (2) to calculate their controls $x_{i}, i=1, \ldots, n$.

The following theorem gives conditions that guarantee attainability of $\overline{\mathbf{z}}=(\overline{\mathbf{x}}, \overline{\mathbf{y}})$ in the environment considered.

Theorem 1. (Nash implementation conditions) Suppose that $f_{j}(\mathbf{x}, \mathbf{y})=\sum_{i=1}^{n} f_{j i}\left(x_{i}\right)+g_{j}(\mathbf{y})$ for $j=$ $0, \ldots, m$. Let $\overline{\mathbf{z}}=(\overline{\mathbf{x}}, \overline{\mathbf{y}})$ be a point for which the second-order necessary optimality conditions for $(\mathrm{P})$ hold with $\operatorname{det} \nabla_{\mathbf{y}} \mathbf{F}(\overline{\mathbf{x}}, \overline{\mathbf{y}}) \neq 0$. If, for $\mathbf{x} \in \mathbb{B}_{\epsilon}(\overline{\mathbf{x}})$, functions $\eta_{i}, i=1, \ldots, n$, are defined by the system

$$
\left\{\begin{array}{l}
\frac{\partial \eta_{i}}{\partial x_{i}}(\mathbf{x})=\sum_{j=1}^{m} p_{j}(\mathbf{x}) \frac{\partial f_{j i}}{\partial x_{i}}\left(x_{i}\right), \quad i=1, \ldots, n \\
\mathbf{p}(\mathbf{x}) \equiv-\left(\frac{\partial \mathbf{F}^{T}}{\partial \mathbf{y}}(\mathbf{x}, \mathbf{Y}(\mathbf{x}))\right)^{-1} \frac{\partial f_{0}}{\partial \mathbf{y}}(\mathbf{x}, \mathbf{Y}(\mathbf{x})) \\
\mathbf{F}(\mathbf{x}, \mathbf{Y}(\mathbf{x})) \equiv 0
\end{array}\right.
$$

and for any $\mathbf{v} \in \mathbb{R}^{n} \backslash\{0\}$ the following condition holds:

$$
\mathbf{v}^{T} \frac{\partial \mathbf{Y}^{T}}{\partial \mathbf{x}}(\overline{\mathbf{x}}) \frac{\partial^{2} H}{\partial \mathbf{y}^{2}}(\overline{\mathbf{x}}, \mathbf{p}(\overline{\mathbf{x}})) \frac{\partial \mathbf{Y}}{\partial \mathbf{x}^{T}}(\overline{\mathbf{x}}) \mathbf{v}>0
$$

where $H(\mathbf{z}, \boldsymbol{\mu})=f_{0}(\mathbf{z})+\boldsymbol{\mu}^{T} \mathbf{F}(\mathbf{z})$, then $\overline{\mathbf{z}}$ is an isolated solution to $(\mathrm{P})$ and also a unique solution to the system

$$
\begin{gathered}
\left(\mathrm{PAYOFF}_{i}, i=1, \ldots, n\right) \\
\begin{cases}\text { minimize } & J_{i}\left(x_{i}, \mathbf{x}_{-i}\right)=f_{0 i}\left(x_{i}\right)+\eta_{i}(\mathbf{x}) \\
\text { over } & x_{i} \in \mathbb{B}_{\epsilon}\left(\bar{x}_{i}\right) .\end{cases}
\end{gathered}
$$

Proof. From the regularity assumption it follows that the set $V(\overline{\mathbf{z}})$ of feasible variations is given by

$$
V(\overline{\mathbf{z}})=\left\{\mathbf{d}(\mathbf{v})=\left(\mathbf{v}, \nabla_{\mathbf{x}} \mathbf{Y}(\overline{\mathbf{x}}) \mathbf{v}\right)^{T} \mid \mathbf{v} \in \mathbb{R}^{n} \backslash\{0\}\right\} .
$$

Indeed, for $\mathbf{d}(\mathbf{v}) \in V(\overline{\mathbf{z}})$ we get

$$
\left(\frac{\partial \mathbf{F}}{\partial \mathbf{x}^{T}}(\overline{\mathbf{z}})+\frac{\partial \mathbf{F}}{\partial \mathbf{y}^{T}}(\overline{\mathbf{z}}) \frac{\partial \mathbf{Y}}{\partial \mathbf{x}^{T}}(\overline{\mathbf{x}})\right) \mathbf{v}=0 .
$$

Since, by definition of $\mathbf{p}(\mathbf{x})$, there holds

$$
\begin{aligned}
& \frac{\partial \mathbf{p}^{T}}{\partial \mathbf{x}}(\overline{\mathbf{x}}) \frac{\partial \mathbf{F}}{\partial \mathbf{x}^{T}}(\overline{\mathbf{x}}, \mathbf{Y}(\overline{\mathbf{x}})) \\
& \quad=\frac{\partial \mathbf{Y}^{T}}{\partial \mathbf{x}}(\overline{\mathbf{x}}) \frac{\partial^{2} H}{\partial \mathbf{y}^{2}}(\overline{\mathbf{x}}, \mathbf{p}(\overline{\mathbf{x}})) \frac{\partial \mathbf{Y}}{\partial \mathbf{x}^{T}}(\overline{\mathbf{x}})
\end{aligned}
$$

from (4) it follows that for $\mathbf{d}\left(\mathbf{e}_{i}\right) \in V(\overline{\mathbf{z}})$

$$
\frac{\partial \mathbf{p}^{T}}{\partial x_{i}}(\overline{\mathbf{x}}) \frac{\partial \mathbf{F}}{\partial x_{i}}(\overline{\mathbf{x}}, \mathbf{Y}(\overline{\mathbf{x}}))>0, \quad i=1, \ldots, n .
$$

As a result, by (3) and the assumption that the second-order necessary conditions hold, $\bar{x}_{i}, i=1, \ldots, n$, satisfies the second-order sufficiency conditions for an isolated optimum of $J_{i}$ :

$$
\begin{aligned}
\frac{\partial J_{i}}{\partial x_{i}}\left(\bar{x}_{i}, \overline{\mathbf{x}}_{-i}\right)= & \frac{\partial f_{0 i}}{\partial x_{i}}\left(\bar{x}_{i}\right)+\sum_{j=1}^{m} p_{j}(\overline{\mathbf{x}}) \frac{\partial f_{j i}}{\partial x_{i}}\left(\bar{x}_{i}\right)=0, \\
\frac{\partial^{2} J_{i}}{\partial\left(x_{i}\right)^{2}}\left(\bar{x}_{i}, \overline{\mathbf{x}}_{-i}\right)= & \frac{\partial^{2} f_{0 i}}{\partial\left(x_{i}\right)^{2}}\left(\bar{x}_{i}\right)+\sum_{j=1}^{m} p_{j}(\overline{\mathbf{x}}) \frac{\partial^{2} f_{j i}}{\partial\left(x_{i}\right)^{2}}\left(\bar{x}_{i}\right) \\
& +\sum_{j=1}^{m} \frac{\partial p_{j}}{\partial x_{i}}(\overline{\mathbf{x}}) \frac{\partial f_{j i}}{\partial x_{i}}\left(\bar{x}_{i}\right)>0 .
\end{aligned}
$$

Furthermore, for every $i=1, \ldots, n$, the function $J_{i}$ is locally strictly convex in $x_{i}$ for every $\mathbf{x}_{-i}$, where $\mathbf{x} \in$ $\mathbb{B}_{\epsilon}(\overline{\mathbf{x}})$.

Consider now the mapping

$$
\begin{aligned}
\mathbf{T} \mathbf{z} \triangleq\left\{\mathbf{x} \in \mathbb{B}_{\epsilon}(\overline{\mathbf{x}}) \mid\right. & \sum_{i=1}^{n} J_{i}\left(x_{i}, \mathbf{z}_{-i}\right) \\
& \left.=\min _{\mathbf{s} \in \mathbb{B}_{\epsilon}(\overline{\mathbf{x}})} \sum_{i=1}^{n} J_{i}\left(s_{i}, \mathbf{z}_{-i}\right)\right\} \subseteq \mathbb{B}_{\epsilon}(\overline{\mathbf{x}}),
\end{aligned}
$$


where $\mathbb{B}_{\epsilon}(\overline{\mathbf{x}})=\mathbb{B}_{\epsilon}\left(\bar{x}_{1}\right) \times \ldots \times \mathbb{B}_{\epsilon}\left(\bar{x}_{n}\right) . \quad$ By the strict convexity of $J_{i}, i=1, \ldots, n, \mathbf{T}$ is an upper semicontinuous mapping of a closed, bounded and convex set $\mathbb{B}_{\epsilon}(\overline{\mathbf{x}})$ into itself. Thus, by the Kakutani theorem (Kakutani, 1941), there exists $\mathbf{x}^{*} \in \mathbf{T} \mathbf{x}^{*}$, a solution to $\left(\mathrm{PAYOFF}_{i}, i=1, \ldots, n\right)$ establishing a Nash equilibrium point (Rosen, 1965; Basar and Olsder, 1999). It can also be seen that $\overline{\mathbf{x}} \in \mathbf{T} \overline{\mathbf{x}}$. Indeed, we have

$$
\min _{\mathbf{s} \in \mathbb{B}_{\epsilon}(\overline{\mathbf{x}})} \sum_{i=1}^{n} J_{i}\left(s_{i}, \overline{\mathbf{x}}_{-i}\right)=\sum_{i=1}^{n} \min _{s_{i} \in \mathbb{B}_{\epsilon}\left(\bar{x}_{i}\right)} J_{i}\left(s_{i}, \overline{\mathbf{x}}_{-i}\right),
$$

and for every $i=1, \ldots, n$,

$$
J_{i}\left(\bar{x}_{i}, \overline{\mathbf{x}}_{-i}\right)<J_{i}\left(s_{i}, \overline{\mathbf{x}}_{-i}\right), \quad s_{i} \in \mathbb{B}_{\epsilon}\left(\bar{x}_{i}\right) .
$$

Finally, from (4) we have

$$
\begin{array}{r}
\frac{\partial g_{0}}{\partial \mathbf{y}}(\overline{\mathbf{y}})+\sum_{j=1}^{m} \bar{\mu}_{j} \frac{\partial g_{j}}{\partial \mathbf{y}}(\overline{\mathbf{y}})=0, \\
\frac{\partial^{2} g_{0}}{\partial \mathbf{y}^{2}}(\overline{\mathbf{y}})+\sum_{j=1}^{m} \bar{\mu}_{j} \frac{\partial^{2} g_{j}}{\partial \mathbf{y}^{2}}(\overline{\mathbf{y}})>0,
\end{array}
$$

which shows that the second-order sufficient optimality conditions hold for $\overline{\mathbf{y}}=\mathbf{Y}(\overline{\mathbf{x}})$. We therefore conclude that $\overline{\mathbf{z}}=(\overline{\mathbf{x}}, \mathbf{Y}(\overline{\mathbf{x}}))$ is an isolated solution to $(\mathrm{P})$ defined by a unique solution to $\left(\mathrm{PAYOFF}_{i}, i=1, \ldots, n\right)$.

By Theorem 1, if Problem (P) has the required separable structure and there exists a regular point $\overline{\mathbf{z}}$ satisfying the second-order necessary optimality conditions, then $\overline{\mathbf{z}}$ is locally reachable as a solution to the system $\left(\mathrm{PAYOFF}_{i}, i=1, \ldots, n\right)$. This solution is also a Nash equilibrium in the noncooperative price-anticipation game with payoffs $J_{i}, i=1, \ldots, n$, parameterized by the price-based coordination instruments $\eta_{i}, i=1, \ldots, n$. (It should be noticed that only a partial derivative of $\eta_{i}$ is defined.) By construction these instruments remain invariant with respect to the first-order variations of dual variables (prices) in the equilibrium. This property maintains compatibility of the first-order optimality conditions for (P) with the first-order optimality conditions for $\left(\mathrm{PAYOFF}_{i}, i=1, \ldots, n\right)$. In other words, in the equilibrium the incentive compatibility constraint is satisfied.

The proof of Theorem 1 is based on the elimination technique (modeling the reasoning of the price-anticipating agents), according to which the constrained problem is reduced to an unconstrained one with $m$ dependent (interaction or basic) variables expressed in terms of $n$ independent (control) variables. As a consequence, the assumption of regularity, $\operatorname{det} \nabla_{\mathbf{y}} \mathbf{F}(\overline{\mathbf{x}}, \overline{\mathbf{y}}) \neq 0$, seems to be indispensable in the setting considered. It allows the construction of

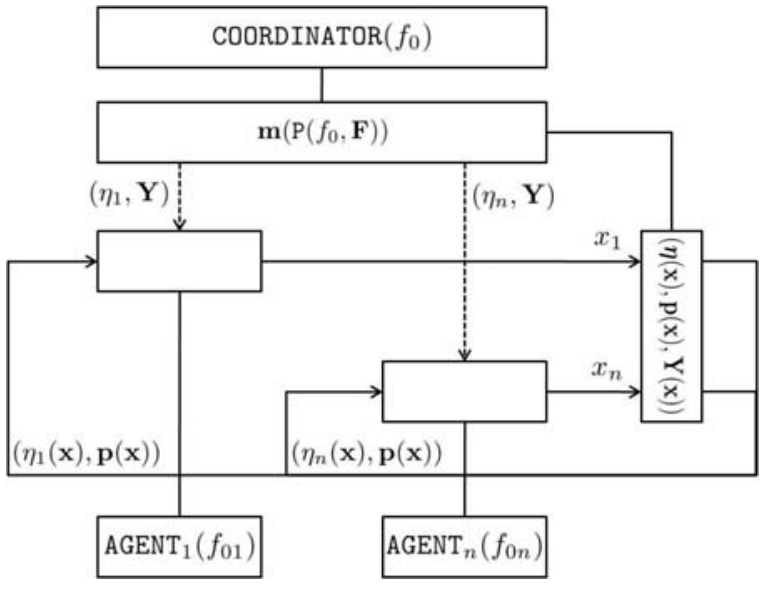

Fig. 2. Implementation scheme of the coordination mechanism.

dual and interaction variables as functions of controls, and thus gives rise to the definition of the analyzed game form. The same can be said about the local strict convexity of the Lagrange function (with respect to the interaction variables). Under the assumption that the first-order optimality conditions hold, the condition (4) implies existence and uniqueness of a solution to $\left(\mathrm{PAYOFF}_{i}, i=1, \ldots, n\right)$. (Nonetheless, it should also be noticed that, if (4) holds with equality, the strict convexity of $f_{0 i}, i=1, \ldots, n$, may be sufficient for the existence of a solution to $\left(\mathrm{PAYOFF}_{i}, i=1, \ldots, n\right)$ as well. This case is illustrated in Section 4 in Example (E3), in which the condition (4) is violated for $\mathbf{y}=\left(z_{1}, z_{4}\right)$.)

We leave it an open question whether it is possible to relax the above conditions. For example, since $\eta_{i}(\overline{\mathbf{x}})=$ $\bar{\eta}_{i}\left(\boldsymbol{\mu}, \bar{x}_{i}\right)$, it may be interesting to investigate the relation between problem $\mathrm{PAYOFF}_{i}$, generalized conjugacy and augmented Lagrangians. Inspirations for further research can be found in the works of Bertsekas and Ozdaglar (2002), Rockafellar and Wets (2004), as well as Jofré et al. (2007).

Mechanism design procedure. Suppose that the coordinator's goal is to optimize the commonly known (utilitarian) system performance indicator, $f_{0}=\sum_{i=1}^{n} f_{0 i}+g_{0}$, aggregating private preference indicators $f_{0 i}, i=1, \ldots, n$, of the agents, subject to the constraints $f_{j}, j=1, \ldots, m$. To reach this goal, the coordinator must design and introduce into the system a mechanism, $\mathbf{m}(\mathrm{P})$, that properly controls interactions of the agents. The following procedure can be applied by the coordinator:

1. Decomposition: Define control variables $\mathbf{x}$ and interaction variables $\mathbf{y}$ for which $\operatorname{det} \nabla_{\mathbf{y}} \mathbf{F}(\mathbf{x}, \mathbf{y}) \neq 0$ and $\mathbf{F}(\mathbf{x}, \mathbf{y})=0$.

Since there may exist many feasible decomposition 
profiles $(\mathbf{x}, \mathbf{y})$, it follows that several mechanisms can be defined in a neighborhood of $\mathbf{F}(\mathbf{x}, \mathbf{y})=0$.

2. Design: For every feasible decomposition $(\mathbf{x}, \mathbf{y})$ determine the rules of interactions of the control variables, $\mathbf{Y}(\mathbf{x})$, such that $\mathbf{F}(\mathbf{x}, \mathbf{Y}(\mathbf{x})) \equiv 0$. Construct functions $\eta_{i}(\mathbf{x}), i=1, \ldots, n$, taking the form of $\mathbf{p}(\mathbf{x})$ into account.

3. Implementation: Introduce into the system the rules of interaction, $\mathbf{Y}(\mathbf{x})$, and assign to each agent $i=$ $1, \ldots, n$ the payment rule $\eta_{i}(\mathbf{x})$ coordinating payoff $J_{i}(\mathbf{x})$.

Following the constructed rules of coordination, the interaction variables $y_{j}, j=1, \ldots, m$, are to be managed by the system coordinator. At the same time, the problem of setting the value of control variable $x_{i}$ is delegated to a designated agent $i=$ $1, \ldots, n$.

Under the assumptions of Theorem 1, in a neighborhood of a solution to $\mathbf{F}(\mathbf{x}, \mathbf{y})=0$ it is possible to design a mechanism $\mathbf{m}(\mathrm{P})$ defined by the interaction balancing rule, $\mathbf{Y}(\mathbf{x})$, and the coordination instrument (price-based penalty function or payment rule), $\boldsymbol{\eta}(\mathbf{x})$. A particular choice of interactions (dependent or basic variables) specifies the coordination structure of the system and, consequently, local properties and the architecture of the equilibrium in a neighborhood of a fixed solution to $\mathbf{F}(\mathbf{x}, \mathbf{Y}(\mathbf{x})) \equiv 0$. The aim of the design procedure is, therefore, to select the rules of interaction that lead the actively competing agents to the desired equilibrium in the most suitable way.

It should be emphasized that $\mathbf{m}(\mathrm{P})$ can be defined only locally for $\mathbf{x} \in \mathbb{B}_{\epsilon}(\overline{\mathbf{x}})$, i.e., in a neighborhood of a point that meets the first-order optimality conditions for (P). Unfortunately, without the additional assumptions on problem convexity, when local information is not global information, this can be viewed as a natural limitation of the design procedure. Since $\overline{\mathrm{x}}$ is unknown a priori, the coordinator must be able to guess the location of the desired solution, $\mathbb{B}_{\epsilon}(\overline{\mathbf{x}})$, and impose bounds on local control variations in advance.

In the case of economic systems, such as auctions, this amounts to imposing additional regulations, for example, taking the form of bidding activity rules (Cramton, 1997; Milgrom, 2004). In the case of automated systems, such as computer networks, application of dedicated protocols and resource allocation rules is required. We can refer to these limitations as equilibrium implementation costs. Clearly, these costs may be substantial and, therefore, may determine the mechanism design decisions. Other related costs are discussed in Section 4 Indeed, it should be pointed out that

$$
\begin{aligned}
\eta_{i}(\overline{\mathbf{x}})= & \sum_{j=1}^{m} \bar{\mu}_{j} f_{j i}\left(\bar{x}_{i}\right) \\
& -\sum_{j=1}^{m} \int_{0}^{\bar{x}_{i}} f_{j i}(s) \mathrm{d} p_{j}\left(s, \overline{\mathbf{x}}_{-i}\right)+b_{i}\left(\overline{\mathbf{x}}_{-i}\right) \\
\neq & \sum_{j=1}^{m} \bar{\mu}_{j} f_{j i}\left(\bar{x}_{i}\right), \quad i=1, \ldots, n .
\end{aligned}
$$

Therefore, without any additional assumptions on $\eta_{i}$ and $b_{i}$, the penalties arising in the equilibrium at the level of local control are not equal to those optimizing locally the Lagrange function $H(\overline{\mathbf{z}}, \overline{\boldsymbol{\mu}})$, i.e., the penalties given by $\sum_{j=1}^{m} \bar{\mu}_{j} f_{j i}\left(\bar{x}_{i}\right), i=1, \ldots, n$. This brings us to the following, somewhat expected conclusion. Even though it is possible to design $\overline{\mathbf{z}}$ as an equilibrium point in the price-anticipation game, one should await that this would entail additional costs of coordination. This problem is further discussed in Section 4

Geometric interpretation of the mechanism rules is illustrated in Fig. 3, in which the following problem is considered:

$$
\begin{cases}\text { minimize } & f_{0}(\mathbf{z})=z_{1}^{2}+2 z_{2}^{2}+3 z_{3}^{2}+z_{4}^{2} \\ \text { subject to } & f_{1}(\mathbf{z})=1-z_{1}-z_{2}+z_{3}=0 \\ & f_{2}(\mathbf{z})=2-z_{1}+z_{2}-z_{3}+z_{4}=0 \\ \text { over } & \mathbf{z} \in \mathbb{R}^{4}\end{cases}
$$

It can be verified that the following decomposition satisfies the assumptions of Theorem 1, $\mathbf{y}=\left(z_{1}, z_{2}\right)$ and $\mathbf{x}=\left(z_{3}, z_{4}\right)$. In the figure the levels of $H(\mathbf{y}, \mathbf{p}(\mathbf{x}))$ are shown on the left over the feasible region in $\left(Y_{1}(\mathbf{x}), Y_{2}(\mathbf{x})\right)$ space. On the right, the levels of $J_{1}\left(x_{1}, x_{2}^{*}\right)+J_{2}\left(x_{2}, x_{1}^{*}\right)$ are shown together with the best response curves in $\left(x_{1}, x_{2}\right)$ space. As the control variables approach optimal solution at $\mathbf{x}^{*}=\mathbf{T} \mathbf{x}^{*}=\left(\bar{z}_{3}, \bar{z}_{4}\right)$, the interaction variables approach optimal solution at $\left(Y_{1}\left(\mathbf{x}^{*}\right), Y_{2}\left(\mathbf{x}^{*}\right)\right)=\left(\bar{z}_{1}, \bar{z}_{2}\right)$ in the region of feasible interactions. Figure 3 illustrates a sequence of mutual best responses (tâtonnement process) that converges to $\overline{\mathbf{z}}$.

Incentive compatibility. The outcome of the design procedure discussed above can be described as an incentive compatible mechanism defining the price-anticipation game with a Nash equilibrium point establishing the coordinator's local goal. Within the addressed setting the incentive compatibility constraint is expressed (in the language of the price-anticipation game) by the condition (3). This important relation describes the family of rules $\eta_{i}, i=1, \ldots, n$, motivating the agents to coordinate their individual and privately known goals on the commonly known goal of the coordinator, expressed by the steady-state condition (1). The 

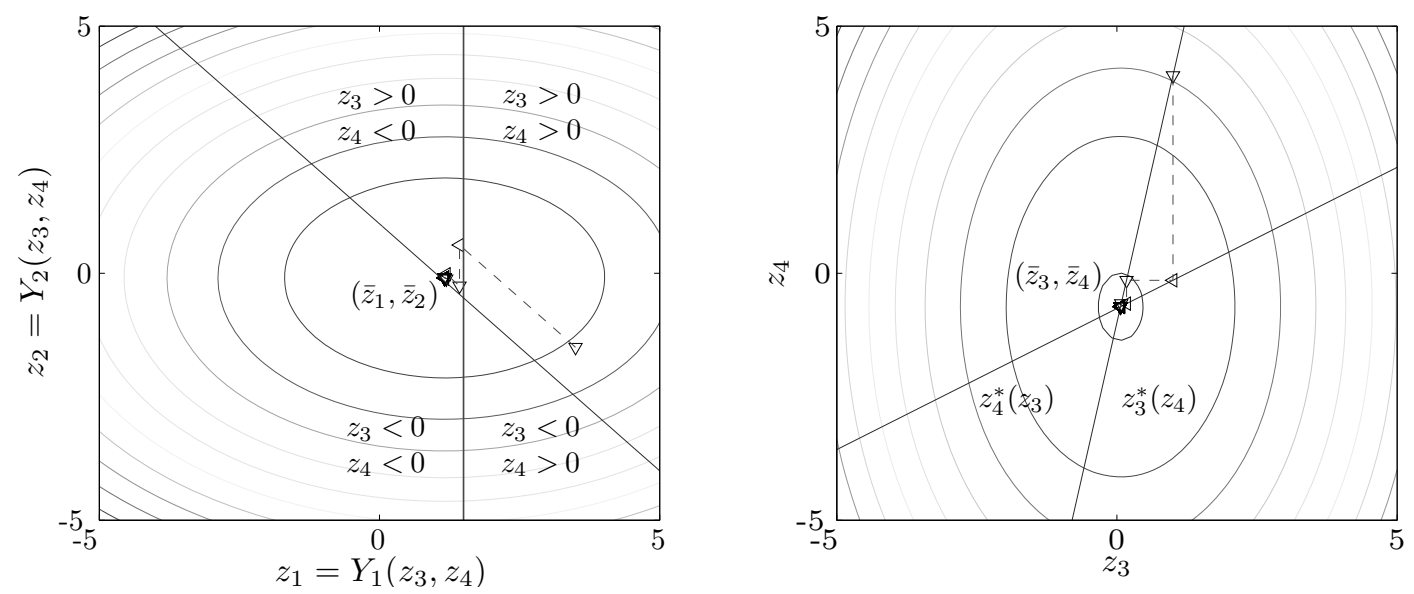

Fig. 3. Geometric interpretation of mechanism rules.

function $\eta_{i}$, by its construction, can be interpreted as a Vickrey-Clarke-Groves transfer (see Mas-Colell et al., 1995, Chapter 23) applied in the studied setting of the price-anticipation game. However, in contrast to standard forms of these transfers, functions $\eta_{i}$ are entirely defined by (2), rather than by the agent's preference indicators. This implies that the agents are not required to reveal to the mechanism the forms of their preference indicators $f_{0 i}, i=1, \ldots, n$, . For the class of mechanisms considered only local characterization of preferences is required, which goes along with the following postulate formulated by Vickrey (1961):

What the $(\ldots)$ agency needs $(. .$.$) is$ an unbiased report of the marginal-cost (competitive supply) curves (...) and of the marginal-value (competitive demand) curves $(\ldots)$, or at least of the portions of these curves covering a range of prices that will be sure to contain the equilibrium price.

Let us point out two important consequences of the incentive compatible implementation of the coordination goal. First, if all the agents apply the price-anticipating strategy to calculate the controls optimizing payoffs $J_{i}, i=1, \ldots, n$, then they realize the goal of coordination (in terms of the first-order optimality conditions) by selfishly acting autonomously in their own best interest. Second, in the Nash equilibrium of the game induced by the rules of $\mathbf{m}(\mathrm{P})$, dual variables are assigned values $\bar{\mu}=\mathbf{p}(\overline{\mathbf{x}})$, equal to those that would be obtained under complete information, i.e., if the coordinator had enough information to perform centralized optimization of $(\mathrm{P})$. This, however, implies that controls optimizing payoffs could be iteratively calculated ignoring the explicit form of $\mathbf{p}(\mathbf{x})$. Namely, since no profitable manipulations of $\mathbf{p}(\mathbf{x})$ (distortions of $\boldsymbol{\mu}=\mathbf{p}(\mathbf{x})$ ) are possible under the rules of coordination applied, it is optimal for the agents to calculate the best responses to the observed values of dual variables $\boldsymbol{\mu}=\mathbf{p}(\mathbf{x})$.

The above conclusions may be useful for the design of iterative procedures that could lead the agents to an equilibrium point under incomplete information. In order to illustrate the general concept, let us again consider the problem $(\mathrm{S})$ discussed in Section 2 . As we have argued above, in the equilibrium the price-taking control is equivalent to the optimal price-anticipating one. Thus, given a vector of prices $\boldsymbol{\mu}(k)$ observed in iteration $k$, each (actively price-anticipating) agent $i$ should solve the problem

$$
\left(\mathrm{A}_{*}^{i}(\boldsymbol{\mu}(k))\right)\left\{\begin{array}{l}
\operatorname{maximize} U_{i}\left(x_{i}\right)-\bar{\eta}_{i}\left(\boldsymbol{\mu}(k), x_{i}\right) \\
\operatorname{over} x_{i} \in \mathbb{B}_{\epsilon}\left(\bar{x}_{i}\right),
\end{array}\right.
$$

where $\mathrm{d} \bar{\eta}_{i}\left(\boldsymbol{\mu}(k), x_{i}\right) / \mathrm{d} x_{i}=\sum_{j \in \mathcal{D}(i)} \mu_{j}(k)$, by (3). Solutions $x_{i}(k), i=1, \ldots, n$, could be then applied to adjust the prices according to the rule $\boldsymbol{\mu}(k+$ $1)=\mathbf{p}(\mathbf{x}(k))$. Obviously, individual allocation costs $\bar{\eta}_{i}\left(\mathbf{p}(\mathbf{x}(k)), x_{i}(k)\right)$ should be adjusted accordingly as well. In Section 5 we illustrate a potential practical application of the above design. A discussion of iterative price-based coordination algorithms for price-anticipating agents can be found in the works of Karpowicz (2010) and Lubacz et al. (2011).

\section{Mechanism design games}

In this section we demonstrate how Theorem 1 can be applied to design and analyze mechanisms that coordinate decisions of price-anticipating agents. Let us emphasize that only for this analytical purpose it is assumed that functions $f_{0 i}, i=1, \ldots, n$, and $f_{j}=1, \ldots, m$ belong to a predefined class known to the mechanism 
designer. Under this assumption, the mechanism design procedure can be used to identify the potential costs of counterspeculation and their determinants.

First, the construction of coordination instruments is discussed and the role played by the conditions (3) and (4) is illustrated. In particular, we show that if the condition (4) is not satisfied, then the Lagrange multipliers may be independent of the control variables. In the price-anticipating environment considered this may be viewed as a potentially desirable property of coordination signals. Next, we turn to the question concerning the costs of introducing the counterspeculative incentives into the system. We illustrate the following phenomenon discovered by Hurwicz (1977):

One is faced with the dilemma of accepting mechanisms that are either not Pareto-satisfactory (with no-trade option) or not privacy-respecting. If one does not wish to sacrifice Pareto-satisfactoriness, one is forced into making concessions on the side of informational decentralization, specifically with regard to privacy. These concessions will involve the diversion of some resources from substantive uses to the operation of the mechanism-to obtaining more information concerning the characteristics of the various individuals, and perhaps, to induce behavior prescribed by the mechanism.

Mechanism design theory shows that for a wide class of problems it is impossible to avoid the costs of Nash equilibrium design (for an overview, see Green and Laffont, 1979; Hurwicz and Walker, 1990; Hurwicz et al., 1995; Mas-Colell et al., 1995; Krishna, 2002). In the general case, gains from reaching a desired solution to the coordination problem need not balance the losses corresponding to the introduction of incentives that make this solution attainable in a noncooperative game. These costs must be incurred by the coordinator through violation of the budget-balancing condition, or the agents through violation of the rational participation constraint. We present how Theorem 1 can be applied to identify and control these costs, at least to some extent, and how the efficiency of design depends on the choice of interaction variables in the system.

The following notation is used below. By $\mathscr{R}(\mathrm{E})$ we denote an optimal solution to problem $(\mathrm{E}), \mathscr{D}_{k l}(\mathrm{E})$ denotes a solution to the price-anticipation game designed for Problem (E) with the interaction variables $\mathbf{y}=$ $\left(z_{k}, z_{l}\right), \mathscr{D}_{k l}^{b}(\mathrm{E})$ denotes a solution to the same game with balanced payoffs, $\mathscr{G}_{k l}(\mathrm{E})$ denotes a solution to (E) with price-anticipating agents and interaction variables $\mathbf{y}=$ $\left(z_{k}, z_{l}\right)$. Finally, for $\mathbf{x}=\left(x_{1}, x_{2}\right)=\left(z_{k}, z_{l}\right)$, we denote $J_{k}=J_{1}(\mathbf{x}), J_{l}=J_{2}(\mathbf{x})$, for $\mathbf{y}=\left(y_{1}, y_{2}\right)=\left(z_{k}, z_{l}\right)$ we write $H_{k}=H_{1}\left(z_{k}, \boldsymbol{\mu}\right), H_{l}=H_{2}\left(z_{l}, \boldsymbol{\mu}\right)$, and $Q=$ $\sum_{i} J_{i}+\sum_{j} H_{j}$. Finally, $\Delta H=|Q-H|$ denotes the implementation cost.

Design of coordination instruments. Consider the following problem due to Fiacco and McCormick (1990):

$$
\text { (E1) } \begin{cases}\text { minimize } & f_{0}\left(z_{1}, z_{2}\right)=\left(z_{1}-1\right)^{2}+z_{2}^{2} \\ \text { subject to } & f_{1}\left(z_{1}, z_{2}\right)=2 \gamma z_{1}-z_{2}^{2} \leq 0 \\ & \gamma>0\end{cases}
$$

There are three solutions of (E1) located on the boundary of feasible set, namely,

$$
\begin{aligned}
& \mathscr{R}(\mathrm{E} 1)= \\
& \left\{\begin{array}{l}
\overline{\mathbf{z}}_{1}=(0,0), \bar{\mu}_{1}=1 / \gamma, \gamma \geq 1 \\
\overline{\mathbf{z}}_{2,3}=(1-\gamma, \pm \sqrt{2 \gamma(1-\gamma)}), \bar{\mu}_{2,3}=1, \gamma \in(0,1)
\end{array}\right.
\end{aligned}
$$

Let us investigate their implementability by following the prescription of Theorem 1

Decomposition of (E1) with respect to $z_{i}, i=1,2$, yields the payoffs

$$
\begin{aligned}
& H_{1}\left(z_{1}, \mu\right)=\left(z_{1}-1\right)^{2}+2 \gamma z_{1} \mu, \\
& H_{2}\left(z_{2}, \mu\right)=z_{2}^{2}(1-\mu),
\end{aligned}
$$

which can be assigned to the agents interacting in the coordinated system. Regularity conditions are satisfied for every $\overline{\mathbf{z}}_{i}, i=1,2,3$. However, the Hessian matrix

$$
\frac{\partial^{2} H}{\partial \mathbf{z}^{2}}(\mathbf{z}, \mu)=\left(\begin{array}{cc}
2 & 0 \\
0 & 2(1-\mu)
\end{array}\right)
$$

of the Lagrange function $H(\mathbf{z}, \mu)=\left(z_{1}-1\right)^{2}+z_{2}^{2}+$ $\mu\left(2 \gamma z_{1}-z_{2}^{2}\right)=H_{1}\left(z_{1}, \mu\right)+H_{2}\left(z_{2}, \mu\right)$ is not positive definite at $\left(\overline{\mathbf{z}}_{1}, \bar{\mu}_{1}\right)$, as required.

There are two cases of interaction variables to be analyzed, $y \triangleq z_{1}$ and $y \triangleq z_{2}$. For each case we get the following outcomes:

$$
\begin{aligned}
& y \triangleq z_{1}, x \triangleq z_{2}: y(x)=x^{2} / 2 \gamma, p(x)=1 / \gamma-x^{2} / 2 \gamma^{2} \\
& y \triangleq z_{2}, x \triangleq z_{1}: y(x)= \pm \sqrt{2 \gamma x}, p(x)=1 .
\end{aligned}
$$

Verify that for $y \triangleq z_{1}$ the assumptions of Theorem 1 hold for every $\overline{\mathbf{z}}_{i}, i=1,2,3$. This implies that every solution to (E1) can be obtained in the decentralized system in which there is an active agent calculating controls $z_{2}$ (determining the value of interaction variable $z_{1}$ ), anticipating the values of the Lagrange multiplier $\mu$. By Theorem 1, the agent must be assigned the payoff

$$
J_{2}\left(z_{2}\right)=z_{2}^{2}-\int_{0}^{z_{2}} 2\left(1 / \gamma-s^{2} / 2 \gamma^{2}\right) s \mathrm{~d} s
$$


Table 1. Implementation outcomes for Problem (E2).

\begin{tabular}{|c|c|c|c|c|c|c|c|c|c|}
\hline & $Q$ & $J_{3}$ & $H_{1}$ & $H_{2}$ & $p_{1}$ & $p_{2}$ & $\Delta H$ & $H+\Delta H$ & $\left\|\overline{\mathbf{z}}-\mathbf{z}^{*}\right\|$ \\
\hline \hline $\mathscr{R}$ & 2.550 & -0.120 & -2.250 & -0.180 & 0.900 & 2.100 & 0.000 & 2.550 & 0.000 \\
$\mathscr{G}_{12}$ & 2.566 & -0.143 & -2.250 & -0.255 & 0.786 & 2.214 & 0.016 & 2.566 & 0.081 \\
$\mathscr{D}_{12}$ & 2.470 & -0.200 & -2.250 & -0.180 & 0.900 & 2.100 & 0.080 & 2.630 & 0.000 \\
\hline & $Q$ & $J_{2}$ & $H_{1}$ & $H_{3}$ & $p_{1}$ & $p_{2}$ & $\Delta H$ & $H+\Delta H$ & $\left\|\overline{\mathbf{z}}-\mathbf{z}^{*}\right\|$ \\
\hline \hline $\mathscr{R}$ & 2.550 & -0.180 & -2.250 & -0.120 & 0.900 & 2.100 & 0.000 & 2.550 & 0.000 \\
$\mathscr{G}_{13}$ & 2.613 & -0.281 & -2.250 & -0.293 & 0.562 & 2.438 & 0.063 & 2.613 & 0.159 \\
$\mathscr{D}_{13}$ & 2.280 & -0.450 & -2.250 & -0.120 & 0.900 & 2.100 & 0.270 & 2.820 & 0.000 \\
\hline
\end{tabular}

derived from the condition (3). Indeed, from the first order optimality conditions we obtain

$$
\mathscr{R}\left(\mathrm{PAYOFF}_{2}\right)=\left\{\begin{array}{l}
z_{2}^{*}=0, \\
z_{2}^{*}= \pm \sqrt{2 \gamma(1-\gamma)},
\end{array}\right.
$$

which yields the following implementation result:

$$
\begin{aligned}
& \mathscr{D}_{1}(\mathrm{E} 1)= \\
& \left\{\begin{array}{c}
z_{2}^{*}=0, y\left(z_{2}^{*}\right)=0, p\left(z_{2}^{*}\right)=1 / \gamma, \gamma \geq 1, \\
z_{2}^{*}= \pm \sqrt{2 \gamma(1-\gamma)}, y\left(z_{2}^{*}\right)=1-\gamma, p\left(z_{2}^{*}\right)=1, \\
0<\gamma<1 .
\end{array}\right.
\end{aligned}
$$

Suppose now that $y \triangleq z_{2}$. It can be verified that in this case $\partial^{2} H(\mathbf{z}, \mu) / \partial y^{2}=2(1-p(x))=0$. Notice that $p(x)=1$, i.e., it does not depend on the control variable $x$. As a result, first-order optimality conditions for

$$
J_{1}\left(z_{1}\right)=\left(z_{1}-1\right)^{2}+\tilde{\mu} \int_{0}^{z_{1}} 2 \gamma \mathrm{d} s
$$

yield

$$
\mathscr{R}\left(\mathrm{PAYOFF}_{1}\right)=\left\{z_{1}^{*}=1-\gamma \tilde{\mu}\right\}
$$

Therefore, $\overline{\mathbf{z}}_{i}, \quad i=1,2,3$, with $\tilde{\mu}=p\left(z_{1}\right)=1$, is reachable only for $0<\gamma \leq 1$. Solutions to $\mathrm{PAYOFF}_{1}$ that are reachable for $\gamma>1$ do not generate the solution $\overline{\mathbf{z}}_{1}$ to (E1). For every $\overline{\mathbf{z}}_{i}, i=1,2,3$, to be determined by $z_{1}$ we must have

$$
\tilde{\mu}= \begin{cases}1, & 0<\gamma \leq 1 \\ 1 / \gamma, & \gamma>1\end{cases}
$$

and $J_{1}=H_{1}$, i.e., the price-taking strategy must be applied by the agent.

Implementation cost. Let us now apply the developed tools to illustrate the costs of introducing into the system the countermeasures preventing the distortion of outcomes under price-anticipating strategies. In particular, we will show that it is not always profitable for the coordinator to impose the incentive compatibility constraints.

Consider the following problem:

$$
(\mathrm{E} 2) \begin{cases}\operatorname{minimize} & f_{0}(\mathbf{z})=z_{1}^{2}+2 z_{2}^{2}+3 z_{3}^{2} \\ \text { subject to } & f_{1}(\mathbf{z})=z_{1}+z_{2}-z_{3}=1, \\ & f_{2}(\mathbf{z})=z_{1}-z_{2}+z_{3}=2, \\ \text { over } & \mathbf{z} \in \mathbb{R}^{3} .\end{cases}
$$

The corresponding Lagrange function $H(\mathbf{z}, \boldsymbol{\mu})=f_{0}(\mathbf{z})+$ $\mu_{1}\left(1-f_{1}(\mathbf{z})\right)+\mu_{2}\left(2-f_{2}(\mathbf{z})\right)$ can be easily decomposed into

$$
\begin{aligned}
& H_{1}\left(z_{1}, \boldsymbol{\mu}\right)=z_{1}^{2}-z_{1}\left(\mu_{1}+\mu_{2}\right), \\
& H_{2}\left(z_{2}, \boldsymbol{\mu}\right)=2 z_{2}^{2}-z_{2}\left(\mu_{1}-\mu_{2}\right), \\
& H_{3}\left(z_{3}, \boldsymbol{\mu}\right)=3 z_{3}^{2}-z_{3}\left(\mu_{2}-\mu_{1}\right) .
\end{aligned}
$$

We therefore have $H(\mathbf{z}, \boldsymbol{\mu})=H_{1}\left(z_{1}, \boldsymbol{\mu}\right)+H_{2}\left(z_{2}, \boldsymbol{\mu}\right)+$ $H_{3}\left(z_{3}, \boldsymbol{\mu}\right)+\left(\mu_{1}+2 \mu_{2}\right)$. It can also be verified that

$$
\mathscr{R}(\mathrm{E} 2)=\left\{\begin{array}{l}
\overline{\mathbf{z}}=(1.5,-.3, .2)^{T}, \\
\overline{\boldsymbol{\mu}}=(.9,2.1)^{T}
\end{array}\right.
$$

where

$$
\mathbf{d}^{T} \frac{\partial^{2} H}{\partial \mathbf{z}^{2}}(\overline{\mathbf{z}}, \overline{\boldsymbol{\mu}}) \mathbf{d}=\mathbf{d}^{T}\left(\begin{array}{ccc}
2 & 0 & 0 \\
0 & 4 & 0 \\
0 & 0 & 6
\end{array}\right) \mathbf{d}>0, \quad \mathbf{d} \neq 0 .
$$

Implementation of the above global solution to (E2) requires that three sets of interaction variables be considered: $\left\{z_{1}, z_{2}\right\},\left\{z_{1}, z_{3}\right\}$ and $\left\{z_{2}, z_{3}\right\}$. Only two of them are feasible, namely

$$
\begin{aligned}
\mathbf{y}=\left(z_{1}, z_{2}\right), \quad x \triangleq z_{3}: \mathbf{Y}(x) & =(1.5, x-.5)^{T}, \\
\mathbf{p}(x) & =(2 x+.5,2.5-2 x)^{T} . \\
\mathbf{y}=\left(z_{1}, z_{3}\right), \quad x \triangleq z_{2}: \mathbf{Y}(x) & =(1.5, x+.5)^{T}, \\
\mathbf{p}(x) & =(-3 x, 3 x+3)^{T} .
\end{aligned}
$$

For the interactions defined by $\left\{z_{2}, z_{3}\right\}$ the regularity condition is violated. Table 2 shows the construction of mechanisms that are feasible for (E2). 
Table 2. Mechanisms designed for Problem (E2).

\begin{tabular}{|c|c|c|c|c|}
\hline & $p_{1}(\mathbf{x})$ & $p_{2}(\mathbf{x})$ & $Y_{1}(\mathbf{x})$ & $Y_{2}(\mathbf{x})$ \\
\hline \hline $\mathscr{D}_{12}$ & $2 z_{3}+0.5$ & $2.5-2 z_{3}$ & 1.5 & $z_{3}-0.5$ \\
$\mathscr{D}_{13}$ & $-3 z_{2}$ & $3 z_{2}+3$ & 1.5 & $z_{2}+0.5$ \\
\hline
\end{tabular}

Table 3. Mechanism design game.

\begin{tabular}{|cccc|}
\hline & & \multicolumn{2}{c|}{ AGENT } \\
& & PA & PT \\
COORDINATOR & a & $2.630,-0.200$ & $2.639,-0.110$ \\
& na & $2.566,-0.143$ & $2.550,-0.120$ \\
\hline
\end{tabular}

Let us first focus on $\mathbf{y}=\left(z_{1}, z_{2}\right)$, with $x \triangleq$ $z_{3}$, and suppose that there is a price-anticipating agent in the system optimizing function $H_{3}$. Therefore, we assume that the system coordinator applies a fixed-price coordination mechanism in the setting in which the active agent applies price-anticipating strategy. Clearly, solution of the problem of minimizing

$$
H_{3}(x, \mathbf{p}(x))=3 x^{2}+x(4 x-2)
$$

yields the following outcome:

$$
\mathscr{G}_{12}(\mathrm{E} 2)=\left\{\begin{array}{l}
\tilde{\mathbf{z}}=(1.5,-.357, .14)^{T}, \\
\tilde{\boldsymbol{\mu}}=(.78,2.21)^{T}
\end{array}\right.
$$

which is not the solution to (E2). In order to implement $\mathscr{R}(\mathrm{E} 2)$ in the setting in which the price-anticipating strategy is applied, the agent must be assigned the subproblem of minimizing

$$
\begin{aligned}
J_{3}(x) & =3 x^{2}+x(4 x-2)-2 x^{2} \\
& =H_{3}(x, \mathbf{p}(x))-h_{3}(x) .
\end{aligned}
$$

Indeed, in such a case it can be demonstrated that

$$
\mathscr{D}_{12}(\mathrm{E} 2)=\mathscr{R}(\mathrm{E} 2)=\left\{\begin{array}{l}
\mathbf{z}^{*}=(1.5,-.3, .2)^{T}, \\
\boldsymbol{\mu}^{*}=(.9,2.1)^{T},
\end{array}\right.
$$

as required.

Let us take a closer look at the values of payoffs that are obtained in each analyzed case, as presented in Table 1 The solution to the problem of fixed-price coordination in which price-anticipating strategy is applied, $\mathscr{G}_{12}(\mathrm{E} 2)$, is characterized by the following property: $H_{3}\left(\tilde{z}_{3}, \mathbf{p}\left(\tilde{z}_{3}\right)\right)=-.14<-.12=H_{3}\left(\bar{z}_{3}, \overline{\boldsymbol{\mu}}\right)$. Thus, it improves the agent's payoff $H_{3}$ in comparison with the solution $\mathscr{R}(\mathrm{E} 2)$. However, at the same time, $H(\tilde{\mathbf{z}}, \tilde{\boldsymbol{\mu}})=2.57>2.55=H(\overline{\mathbf{z}}, \overline{\boldsymbol{\mu}})$, which means that the price-anticipating decision $\tilde{z}_{3}$ reduces total performance $H$ of the system.
Consider now the case in which Problem $\left(\mathrm{PAYOFF}_{3}\right)$ is solved. By Theorem 1 we get $\mathscr{R}(\mathrm{E} 2)=\mathscr{D}_{12}(\mathrm{E} 2)$. However, this does not imply that the same payoffs are reached in both cases. The obtained results show that introduction of the price-anticipation countermeasures, defined by the incentive compatibility constraint (3), rises the operational cost of the system. This cost is given by the component $h_{3}\left(z_{3}^{*}\right)=0.08$. In the setting considered the optimal system performance level, $H(\overline{\mathbf{z}}, \overline{\boldsymbol{\mu}})$, can only be reached if the price-anticipating agent is able to improve his individual performance in comparison with the level reachable in $\overline{\mathbf{z}}$, i.e., $J_{3}\left(z_{3}^{*}\right)=H_{3}\left(z_{3}^{*}, \mathbf{p}\left(z_{3}^{*}\right)\right)-$ $h_{3}\left(z_{3}^{*}\right)=-0.2<-0.12=H_{3}\left(\bar{z}_{3}, \overline{\boldsymbol{\mu}}\right)$. Therefore, if only the gains generated within the system can be distributed (exchanged) between the agents, then the external transfer equal to $h_{3}\left(z_{3}^{*}\right)$ is required to balance the payoffs, or to satisfy the budget-balancing condition. Consequently, $H(\mathscr{R}(\mathrm{E} 2))=2.55<2.63=H\left(\mathscr{D}_{12}(\mathrm{E} 2)\right)+h_{3}\left(z_{3}^{*}\right)$. Table 1 illustrates attainable costs, denoted by $\Delta H$, of equilibrium design for Problem (E2).

By the above arguments, it is not always reasonable for the coordinator to introduce the incentive compatibility constraints. The following model of the equilibrium design game provides a justification for this argument. Table 3 presents the normal form of the (complete knowledge) game played between the coordinator and the agent capable of making price-anticipating decisions. On the one hand, there are two decisions that may be considered by the coordinator: introduce $a$, and not to introduce, na, the incentives into the system. On the other hand, the agent may decide to be price-anticipating, PA, or price-taking, PT. There exists a unique pure-strategy Nash equilibrium point of the game established by the pair of decisions (na,PA). Therefore, as we have already argued, it is better for the coordinator to reach the suboptimal solution with the price-anticipating agent than the optimal one imposing the incentive compatibility costs. (Payoffs corresponding to $(\mathrm{a}, \mathrm{PT})$ were calculated under the assumption that the agent minimizes $J_{3}\left(z_{3}\right)=3 z_{3}^{2}+z_{3}\left(\mu_{1}-\mu_{2}\right)-2 z_{3}^{2}$.) 
Table 4. Implementation outcomes for Problem (E3).

\begin{tabular}{|c|c|c|c|c|c|c|c|c|c|c|}
\hline & $Q$ & $J_{3}$ & $J_{4}$ & $H_{1}$ & $\mathrm{H}_{2}$ & $p_{1}$ & $p_{2}$ & $\Delta H$ & $H+\Delta H$ & $\left\|\overline{\mathbf{z}}-\mathbf{z}^{*}\right\|$ \\
\hline $\mathscr{R}$ & 1.839 & -0.012 & -0.459 & -1.349 & -0.019 & 0.968 & 1.355 & 0.000 & 1.839 & 0.000 \\
\hline $\mathscr{G}_{12}$ & 1.910 & -0.040 & -0.552 & -1.601 & -0.072 & 0.886 & 1.644 & 0.071 & 1.910 & 0.250 \\
\hline $\mathscr{D}_{12}$ & 1.486 & -0.021 & -0.803 & -1.349 & -0.019 & 0.968 & 1.355 & 0.352 & 2.191 & 0.000 \\
\hline \multirow[t]{2}{*}{$\mathscr{D}_{12}^{b}$} & 1.839 & 0.323 & -0.795 & -1.349 & -0.019 & 0.968 & 1.355 & 0.000 & 1.839 & 0.000 \\
\hline & $Q$ & $J_{2}$ & $J_{4}$ & $H_{1}$ & $H_{3}$ & $p_{1}$ & $p_{2}$ & $\Delta H$ & $H+\Delta H$ & $\left|\overline{\mathbf{z}}-\mathbf{z}^{*}\right| \mid$ \\
\hline $\mathscr{R}$ & 1.839 & -0.019 & -0.459 & -1.349 & -0.012 & 0.968 & 1.355 & 0.000 & 1.839 & 0.000 \\
\hline $\mathscr{G}_{13}$ & 1.939 & -0.086 & -0.603 & -1.628 & -0.089 & 0.759 & 1.793 & 0.101 & 1.939 & 0.278 \\
\hline $\mathscr{D}_{13}$ & 1.352 & -0.047 & -0.918 & -1.349 & -0.012 & 0.968 & 1.355 & 0.487 & 2.326 & 0.000 \\
\hline \multirow[t]{2}{*}{$\mathscr{D}_{13}^{b}$} & 1.839 & 0.412 & -0.890 & -1.349 & -0.012 & 0.968 & 1.355 & 0.000 & 1.839 & 0.000 \\
\hline & $Q$ & $J_{2}$ & $J_{3}$ & $H_{1}$ & $H_{4}$ & $p_{1}$ & $p_{2}$ & $\Delta H$ & $H+\Delta H$ & $\left\|\overline{\mathbf{z}}-\mathbf{z}^{*}\right\|$ \\
\hline $\mathscr{R}$ & 1.839 & -0.019 & -0.012 & -1.349 & -0.459 & 0.968 & 1.355 & 0.000 & 1.839 & 0.000 \\
\hline $\mathscr{G}_{14}$ & 1.853 & -0.045 & -0.037 & -1.242 & -0.594 & 0.687 & 1.542 & 0.014 & 1.853 & 0.111 \\
\hline $\mathscr{D}_{14}$ & 1.771 & -0.066 & -0.033 & -1.349 & -0.459 & 0.968 & 1.355 & 0.068 & 1.906 & 0.000 \\
\hline \multirow[t]{2}{*}{$\mathscr{D}_{14}^{b}$} & 1.839 & -0.045 & 0.014 & -1.349 & -0.459 & 0.968 & 1.355 & 0.000 & 1.839 & 0.000 \\
\hline & $Q$ & $J_{1}$ & $J_{3}$ & $\mathrm{H}_{2}$ & $H_{4}$ & $p_{1}$ & $p_{2}$ & $\Delta H$ & $H+\Delta H$ & $\left\|\overline{\mathbf{z}}-\mathbf{z}^{*}\right\|$ \\
\hline $\mathscr{R}$ & 1.839 & -1.349 & -0.012 & -0.019 & -0.459 & 0.968 & 1.355 & 0.000 & 1.839 & 0.000 \\
\hline $\mathscr{G}_{24}$ & 3.819 & -4.644 & -0.092 & -0.165 & -3.257 & 4.759 & 3.609 & 1.980 & 3.819 & 1.330 \\
\hline $\mathscr{D}_{24}$ & -6.261 & -9.440 & -0.021 & -0.019 & -0.459 & 0.968 & 1.355 & 8.100 & 9.939 & 0.000 \\
\hline \multirow[t]{2}{*}{$\mathscr{D}_{24}^{b}$} & 1.839 & -9.432 & 8.071 & -0.019 & -0.459 & 0.968 & 1.355 & 0.000 & 1.839 & 0.000 \\
\hline & $Q$ & $J_{1}$ & $J_{2}$ & $H_{3}$ & $H_{4}$ & $p_{1}$ & $p_{2}$ & $\Delta H$ & $H+\Delta H$ & $\left\|\overline{\mathbf{z}}-\mathbf{z}^{*}\right\|$ \\
\hline $\mathscr{R}$ & 1.839 & -1.349 & -0.019 & -0.012 & -0.459 & 0.968 & 1.355 & 0.000 & 1.839 & 0.000 \\
\hline $\mathscr{G}_{34}$ & 4.072 & -4.832 & -0.210 & -0.219 & -3.478 & 5.351 & 3.730 & 2.233 & 4.072 & 1.393 \\
\hline $\mathscr{D}_{34}$ & -7.630 & -10.789 & -0.047 & -0.012 & -0.459 & 0.968 & 1.355 & 9.468 & 11.307 & 0.000 \\
\hline $\mathscr{D}_{34}^{b}$ & 1.839 & -10.761 & 9.393 & -0.012 & -0.459 & 0.968 & 1.355 & 0.000 & 1.839 & 0.000 \\
\hline
\end{tabular}

Design of implementation costs. Let us now consider the problem

$$
\text { (E3) } \begin{cases}\text { minimize } & f_{0}(\mathbf{z})=z_{1}^{2}+2 z_{2}^{2}+3 z_{3}^{2}+z_{4}^{2} \\ \text { subject to } & f_{1}(\mathbf{z})=1-z_{1}-z_{2}+z_{3}=0, \\ & f_{2}(\mathbf{z})=2-z_{1}+z_{2}-z_{3}+z_{4}=0, \\ \text { over } & \mathbf{z} \in \mathbb{R}^{4} .\end{cases}
$$

Table 4 illustrates the implementation costs, denoted by $\Delta H$, and the agents' payoffs corresponding to the feasible definitions of interaction variables. The following conclusion can be drawn from this study: efficiency of equilibrium design can be controlled, at least to some extent, by a proper decomposition of the coordination problem. Indeed, by Theorem 1, a particular definition of interactions of the control variables, $\mathbf{Y}(\mathbf{x})$, specifies local properties of the equilibrium point. This observation may be given the following (naive or intuitive) economic interpretation. In order to improve the efficiency of competition in the addressed price-anticipating environment, the market regulator should properly select the set of regulated commodities.

Let us now show that under suitable conditions the implementation costs can also be redistributed among the agents. If $n>1$ and it is possible to construct functions $b_{i}\left(\mathbf{x}_{-i}\right), i=1, \ldots, n$, such that

$$
\sum_{i=1}^{n} b_{i}\left(\mathbf{x}_{-i}\right)=\sum_{i=1}^{n} \sum_{j=1}^{m} \int_{0}^{x_{i}} f_{j i}(s) \mathrm{d} p_{j}\left(s, \mathbf{x}_{-i}\right),
$$

then for the coordination instruments

$$
\begin{aligned}
\eta_{i}\left(x_{i}, \mathbf{x}_{-i}\right) \triangleq & \sum_{j=1}^{m} \int_{0}^{x_{i}} p_{j}\left(s, \mathbf{x}_{-i}\right) \mathrm{d} f_{j i}(s) \\
& +b_{i}\left(\mathbf{x}_{-i}\right)
\end{aligned}
$$

we get the balancing condition

$$
\sum_{i=1}^{n} \eta_{i}\left(x_{i}, \mathbf{x}_{-i}\right)=\sum_{i=1}^{n} \sum_{j=1}^{m} p_{j}(\mathbf{x}) f_{j i}\left(x_{i}\right),
$$


as required.

Consider the collection of mechanisms implementing the global solution to (E3), presented in Table 5 Let us take the decomposition defined by $\mathbf{y}=\left(z_{1}, z_{2}\right)$ and $\mathbf{x}=\left(z_{3}, z_{4}\right)$. Since $f_{j}$ and $p_{j}$ are affine functions, it is possible to design the following balancing rules:

$$
b_{i}\left(x_{k}\right)=0.5 x_{k}^{2} \sum_{j=1}^{2} a_{j k} \frac{\partial p_{j}}{\partial x_{k}}\left(x_{k}\right), \quad i, k \in\{1,2\}, i \neq k .
$$

The required payoffs in the equilibrium are given by

$$
\begin{aligned}
J_{3}\left(z_{3}^{*}, z_{4}^{*}\right) & =H_{3}\left(z_{3}^{*}, \mathbf{p}\left(z_{3}^{*}, z_{4}^{*}\right)\right)-2\left(z_{3}^{*}\right)^{2}+.75\left(z_{4}^{*}\right)^{2} \\
& =.32>-.012=H_{3}\left(z_{3}^{*}, \mathbf{p}\left(z_{3}^{*}, z_{4}^{*}\right)\right) \\
J_{4}\left(z_{4}^{*}, z_{3}^{*}\right) & =H_{4}\left(z_{4}^{*}, \mathbf{p}\left(z_{3}^{*}, z_{4}^{*}\right)\right)-.75\left(z_{4}^{*}\right)^{2}+2\left(z_{3}^{*}\right)^{2} \\
& =-.79<-.46=H_{4}\left(z_{4}^{*}, \mathbf{p}\left(z_{3}^{*}, z_{4}^{*}\right)\right) .
\end{aligned}
$$

As can be easily noticed, with the coordination instruments defined as above the cost of equilibrium design is covered by the payoffs of price-anticipating agents: $J_{3}\left(z_{3}^{*}, z_{4}^{*}\right)+J_{4}\left(z_{4}^{*}, z_{3}^{*}\right)=H_{3}\left(z_{3}^{*}, \mathbf{p}\left(z_{3}^{*}, z_{4}^{*}\right)\right)+$ $H_{4}\left(z_{4}^{*}, \mathbf{p}\left(z_{3}^{*}, z_{4}^{*}\right)\right)$. There are no external transfers required to coordinate the agents' controls. In the equilibrium the required transfers are provided by the agents themselves. This, however, immediately implies that there is a trade-off that cannot be fully eliminated. Introduction of the above balanced coordination rules may discourage the agents from playing the game. Namely, if functions $b_{i}\left(\mathbf{x}_{-i}\right)$ are used, then the participation (or individual rationality) constraints

$$
J_{i}\left(x_{i}^{*}, \mathbf{x}_{-i}^{*}\right) \leq J_{i}^{0}, \quad i=1, \ldots, n,
$$

may be violated in the equilibrium. Table 4 illustrates outcomes that are reachable in the balanced equilibrium under the assumption that the condition (6) is not taken into account by the agents optimizing their control variables. Notice that differences between the balanced and unbalanced payoffs may be substantial and cannot be omitted in the analysis of the mechanism design game. Furthermore, the results clearly show that counterspeculation may be an option for the coordinator only if the balancing condition can be satisfied.

The following interesting regularity can also be observed in Table 4. More profitable incentives are received by this one of a pair of competing agents whose marginal preference indicator, $f_{0 i}^{\prime}\left(x_{i}\right)$, is lower. This suggest that the analysis of equilibrium design costs, as well as the mechanism design procedure, should take into account the relation between the intensity of the agents' preferences, if it is measurable and comparable (Sen, 1970; 1977). In some cases it may be reasonable for the coordinator to allow the competition only between the agents characterized by similar preferences. In fact, this conclusion seems to be compliant with what can be observed in practice. For example, entry fees and reserve prices applied in auction design can be viewed as means of conducting the qualifying process; see the work of Milgrom and Weber (1982) for an extensive study of outcomes arising in auctions with a reserve price and entry fee. Classical results of auction theory can be found in the contribution by Krishna (2002).

\section{Traffic rate control in the Internet}

To illustrate the potential impact of the results presented above we will discuss the design and the properties of the TCP traffic rate (or congestion) control algorithm commonly used in communication networks (for introduction, see Stallings, 1998; Chao and Guo, 2002).

The TCP congestion control algorithm is required to adapt the traffic rate, i.e., the rate at which a TCP node sends packets, to the locally observed congestion signals, usually related to the rate of incoming acknowledgment (ACK) messages. These congestion signals provide information about the utilization of the links in the network and can be interpreted as link capacity prices. It is, therefore, convenient to interpret TCP as a distributed mechanism that optimizes the network performance subject to the link capacity constraints.

In order to engineer a stable and efficient process of transmission rate adaptation, the following procedure is usually applied. First, a reference point is defined that represents preferred outcomes of the resource allocation problem. The reference point is defined by the optimality conditions satisfied by a solution to a suitably formulated optimization problem. Second, based on the specified optimality conditions a dynamic system is constructed that is guaranteed to converge to a neighborhood of the reference point. The definition of the dynamic system is then used as a design guideline for the algorithms implemented in the network.

The problem that underlies current designs, mostly due to Kelly (1997) and Kelly et al. (1998), is defined as follows:

$$
\operatorname{System}(\mathbf{U}, \mathbf{A}, \mathbf{c}) \begin{cases}\operatorname{maximize} & \sum_{i=1}^{n} U_{i}\left(x_{i}\right) \\ \text { subject to } & \mathbf{A}^{T} \mathbf{x} \leq \mathbf{c} \\ \text { over } & x_{i} \geq 0, i=1, \ldots, n .\end{cases}
$$

In the above formulation the routing matrix $\mathbf{A}$ consists of elements $A_{i j}=1$ if Link $j$ is in User $i$ 's route $\mathcal{D}(i)$ and $A_{i j}=0$ otherwise. Elements of vector $\mathbf{c}=\left(c_{1}, \ldots, c_{m}\right)$ denote the finite capacity of links in the network. Notice that specification of the utility functions $U_{i}, i=1, \ldots, n$, is not available due to decentralized nature of the network. It should also be clear that we are dealing with the problem of designing a coordination mechanism m(SYSTEM). 
Table 5. Mechanisms designed for Problem (E3).

\begin{tabular}{|c|c|c|c|c|c|c|}
\hline & $p_{1}(\mathbf{x})$ & $p_{2}(\mathbf{x})$ & $Y_{1}(\mathbf{x})$ & $Y_{2}(\mathbf{x})$ & $b_{1}\left(x_{2}\right)$ & $b_{2}\left(x_{1}\right)$ \\
\hline \hline $\mathbf{y}=\left(z_{1}, z_{2}\right)$ & $2 z_{3}-0.5 z_{4}+0.5$ & $-2 z_{3}+1.5 z_{4}+2.5$ & $0.5 z_{4}+1.5$ & $z_{3}-0.5 z_{4}-0.5$ & $0.75 z_{4}^{2}$ & $2 z_{3}^{2}$ \\
$\mathbf{y}=\left(z_{1}, z_{3}\right)$ & $-3 z_{2}-z_{4}$ & $3 z_{2}+2 z_{4}+3$ & $0.5 z_{4}+1.5$ & $z_{2}+0.5 z_{4}+0.5$ & $z_{4}^{2}$ & $3 z_{2}^{2}$ \\
$\mathbf{y}=\left(z_{1}, z_{4}\right)$ & $6 z_{3}-6 z_{2}$ & $4 z_{2}-4 z_{3}+2$ & $z_{3}-z_{2}+1$ & $2 z_{3}-2 z_{2}-1$ & $5 z_{3}{ }^{2}$ & $5 z_{2}{ }^{2}$ \\
$\mathbf{y}=\left(z_{2}, z_{4}\right)$ & $4 z_{3}-8 z_{1}+10$ & $6-4 z_{1}$ & $z_{3}-z_{1}+1$ & $2 z_{1}-3$ & $2 z_{3}^{2}$ & $6 z_{1}^{2}$ \\
$\mathbf{y}=\left(z_{3}, z_{4}\right)$ & $12-6 z_{2}-10 z_{1}$ & $6-4 z_{1}$ & $z_{1}+z_{2}-1$ & $2 z_{1}-3$ & $3 z_{2}^{2}$ & $7 z_{1}^{2}$ \\
\hline
\end{tabular}

Suppose that each network user may submit to the network a bid $\theta_{i} \geq 0$ denoting willingness to pay for the traffic rate $x_{i}=\theta_{i} / \lambda_{i} \geq 0$, where $\lambda_{i} \geq 0$ can be regarded as a charge per unit traffic flow for user $i$. Let us also assume that each user $i$, taking $\lambda_{i}=\sum_{j=1}^{m} A_{i j} \mu_{j}>0$ as given, chooses $\theta_{i}$ that solves the problem

$$
\operatorname{USER}_{i}\left(U_{i}, \lambda_{i}\right) \text { maximize } U_{i}\left(\frac{\theta_{i}}{\lambda_{i}}\right)-\theta_{i} \text { over } \theta_{i} \geq 0 \text {. }
$$

Next, suppose that, given the vector $\boldsymbol{\theta}=\left(\theta_{1}, \ldots, \theta_{n}\right)$ of bids, the network sets prices (Lagrange multipliers) $\mu_{j} \geq$ $0, j=1, \ldots, m$, and rates $x_{i} \geq 0, i=1, \ldots, n$, solving the problem

$$
\operatorname{NETWORK}(\mathbf{A}, \mathbf{c}, \boldsymbol{\theta}) \begin{cases}\text { maximize } & \sum_{i=1}^{n} \theta_{i} \log \left(x_{i}\right) \\ \text { subject to } & \mathbf{A}^{T} \mathbf{x} \leq \mathbf{c} \\ \text { over } & x_{i} \geq 0, i=1, \ldots, n .\end{cases}
$$

Proposition 2, presented below, shows that under the assumption that the users are price-takers a feasible solution arbitrarily close to the solution of $\operatorname{SYSTEM}(\mathbf{U}, \mathbf{A}, \mathbf{c})$ can be found by a distributed algorithm solving $\operatorname{NETWORK}(\mathbf{A}, \mathbf{c}, \boldsymbol{\theta}(t))$ at time $t$ and, on a larger time scale, driving $\boldsymbol{\theta}(t)$ to $\overline{\boldsymbol{\theta}}$ defining an optimal solution to $\operatorname{SYSTEM}(\mathbf{U}, \mathbf{A}, \mathbf{c})$.

Theorem 2. (Equilibrium decentralization conditions (Kelly, 1997)) Suppose that $U_{i}$ is an increasing, strictly concave and continuously differentiable function over $x_{i} \geq 0$ for $i=1, \ldots, n$. There exist vectors $\bar{\lambda}=$ $\left(\bar{\lambda}_{1}, \ldots, \bar{\lambda}_{n}\right), \overline{\boldsymbol{\theta}}=\left(\bar{\theta}_{1}, \ldots, \bar{\theta}_{n}\right)$ and $\overline{\mathbf{x}}=\left(\bar{x}_{1}, \ldots, \bar{x}_{n}\right)$ such that

- $\bar{\theta}_{i}$ solves $\operatorname{USER}_{i}\left(U_{i}, \bar{\lambda}_{i}\right), i=1, \ldots, n$;

- $\overline{\mathbf{x}}$ solves $\operatorname{NETWORK}(\mathbf{A}, \mathbf{c}, \overline{\boldsymbol{\theta}})$;

- $\bar{\theta}_{i}=\bar{\lambda}_{i} \bar{x}_{i}, i=1, \ldots, n$.

The vector $\overline{\mathbf{x}}$ then also solves $\operatorname{SYSTEM}(\mathbf{U}, \mathbf{A}, \mathbf{c})$.

Based on the above result the distributed rate control algorithm can be derived from the following system of differential equations:

$$
\left\{\begin{array}{c}
\operatorname{RATE}(\mathbf{A}, \mathbf{q}, \boldsymbol{\theta}) \\
\frac{\mathrm{d} x_{i}}{\mathrm{~d} t}(t)=\kappa\left[\theta_{i}-x_{i}(t) \sum_{j} A_{i j} \mu_{j}(t)\right], i=1, \ldots, n, \\
\mu_{j}(t)=q_{j}\left(\sum_{i} A_{i j} x_{i}(t)\right), j=1, \ldots, m .
\end{array}\right.
$$

System $\operatorname{RATE}(\mathbf{A}, \mathbf{q}, \boldsymbol{\theta})$ describes the network-wide traffic rate adjustment process, $x_{i}(t), i=1, \ldots, n$, with feedback provided to each network node by the congestion signals $\mu_{j}(t), j=1, \ldots, m$. By construction, it exploits the properties of an equilibrium point, described in Theorem 2, applying the function $\theta_{i} \log \left(x_{i}\right)$ as a model of User $i$ 's preference indicator. Furthermore, as can be noticed, we are indeed dealing with a uniform-price-based coordination mechanism.

For a fixed signal $\theta_{i}, i=1, \ldots, n$, a well-behaved and suitably designed function $q_{j}, j=1, \ldots, m$, (not necessarily differentiable), system $\operatorname{RATE}(\mathbf{A}, \mathbf{q}, \boldsymbol{\theta})$ can be proved to converge to the point minimizing the function

$$
\sum_{j=1}^{m} \int_{0}^{y} q_{j}(s) \mathrm{d} s-\sum_{i=1}^{n} \theta_{i} \log x_{i}, \quad y=\sum_{i=1}^{n} A_{i j} x_{i} .
$$

Suppose next that each user $i$ updates signals $\theta_{i}$ according to the following price-taking control rule:

$$
\theta_{i}(t)=x_{i}(t) U_{i}^{\prime}\left(x_{i}(t)\right), \quad i=1, \ldots, n .
$$

It can be demonstrated that with $\boldsymbol{\theta}(t)$ evolving as above the rates $\mathbf{x}(t)$ converge to a stable point $\overline{\mathbf{x}}$ minimizing

$$
\sum_{j=1}^{m} \int_{0}^{y} q_{j}(s) \mathrm{d} s-\sum_{i=1}^{n} U_{i}\left(x_{i}\right), \quad y=\sum_{i=1}^{n} A_{i j} x_{i} .
$$

Since for a suitable choice of $q_{j}, j=1, \ldots, m$, the above function arbitrarily closely approximates objective in $\operatorname{SYSTEM}(\mathbf{U}, \mathbf{A}, \mathbf{c})$, it follows that $\overline{\mathbf{x}}$ solves relaxation of the problem. Details concerning practical implementations of the above algorithm can be found in the works of Low and Lapsley (1999), La and Anantharam (2000), Mo and Walrand (2000), Malinowski (2002), Low et al. (2002; 2003), Low (2003), Srikant (2003), and Wei et al. (2006). Clempner and Poznyak (2011) provide a discussion of best-reply dynamics in noncooperative games. 
Price-anticipating congestion control. Since the equilibrium conditions, desired by the coordinator, are built into the congestion control protocol, they are usually commonly known to the network users. On the other hand, the network users are autonomous in choosing their protocol implementations and modifying the parameters of the rate control algorithm. In other words, there exists information asymmetry between the coordinator and the network users, which creates incentives to apply the price-anticipating strategies.

To act as a price-anticipating agent, the network user may modify the rule according to which $\theta_{i}(t)$ is updated. For example, the user may submit

$$
\theta_{i}(t)=x_{i}(t) \tilde{U}_{i}^{\prime}\left(x_{i}(t)\right),
$$

where $\tilde{U}_{i}^{\prime}\left(x_{i}(t)\right)<U_{i}^{\prime}\left(x_{i}(t)\right)$.

Another possible modification sets

$$
\theta_{i}(t)=\tilde{x}_{i}(t) \lambda_{i}(t)
$$

where $\tilde{x}_{i}(t)$ maximizes payoff function $U_{i}\left(x_{i}\right)$ $x_{i} \sum_{j=1}^{m} \int_{0}^{y_{j}} A_{i j} q_{j}(s) \mathrm{d} s$, with $y_{j}=\sum_{k=1}^{n} A_{k j} x_{k}$ estimated by the user. Details concerning the design of the above price-anticipating bidding strategies are given by Karpowicz (2010). Johari (2004) presents a study of (uniform-price) mechanisms generating outcomes with bounded loss of efficiency.

The idea which underlies the construction of the above price-anticipating control rules is simple: the user should reveal to the network a reduced level of demand. Since the network is required to satisfy the observed demand for traffic, its reduced level may give rise to a low price equilibrium in which some of the price-anticipating users receive improved payoffs or traffic rates. A survey of results on demand reduction problem is provided by Krishna (2002), Milgrom (2004), and Karpowicz (2011).

Counterspeculation. In order to reduce the potentially adverse effects of price-anticipation, the coordinator may find it reasonable to implement a modified version to the rate control protocol. Let us discuss a concept of such a design by considering the FAST TCP congestion control algorithm (see, e.g., Jin et al., 2005).

To control the rate at which packets are transmitted FAST TCP algorithm updates the congestion window $w_{i} \geq 0$, i.e., the amount of data the sender can transmit into the network at a given time, based on the observed average round-trip time and average queuing delay. Precisely, each traffic source $i=1, \ldots, n$, adapts $w_{i}$ according to the following rule:

$$
w_{i}(t+1)=\gamma\left(\frac{d_{i} w_{i}(t)}{d_{i}+\lambda_{i}(t)}+\theta_{i}\right)+(1-\gamma) w_{i}(t),
$$

where $d_{i} \geq 0$ denotes the round-trip propagation delay, $\lambda_{i} \geq 0$ denotes the round-trip queuing delay for source $i$ and $\gamma \in(0,1]$. Under some reasonable conditions, already mentioned above, the algorithm can be proved to converge to

$$
\bar{w}_{i}=\bar{\theta}_{i}+\bar{x}_{i} d_{i}, \quad \bar{\theta}_{i}=\bar{x}_{i} \bar{\lambda}_{i}, \quad i=1, \ldots, n .
$$

Thus, in simple terms, in the equilibrium point source $i$ maintains $\bar{\theta}_{i}=\bar{x}_{i} \bar{\lambda}_{i}$ packets in the buffers along its path and $\bar{x}_{i} d_{i}$ packets in the transmission lines (Low et al., 2002; Wei et al., 2006; Mo and Walrand, 2000).

Suppose now that the network users may apply the price-anticipating strategy to adapt their congestion windows. By Theorem 1 and the arguments given in Section 3, under the proper definition of link pricing function $q_{j}, j=1, \ldots, m$, a solution $\mathbf{x}^{*}$ in a neighborhood of (8) can still be reached by the agents adapting $w_{i}$ according to (7). For this to be possible under the assumption that the agents are price-anticipating, the network must provide to the users the rate control incentives given by

$$
\begin{aligned}
& \bar{\eta}_{i}\left(\lambda_{i}, x_{i}\right)=x_{i} \lambda_{i}-\bar{h}_{i}\left(x_{i}\right), \quad i=1, \ldots, n, \\
& \bar{h}_{i}\left(x_{i}\right)=\sum_{j=1}^{m} \int_{0}^{x_{i}} A_{i j} s \mathrm{~d} q_{j}\left(s+\sum_{k \neq i} A_{k j} x_{k}\right)-b_{i}\left(\mathbf{x}_{-i}\right),
\end{aligned}
$$

where $\lambda_{i}=\sum_{j=1}^{m} A_{i j} q_{j}\left(y_{j}\right), \quad i=1, \ldots, n$, and $b_{i}\left(\mathbf{x}_{-i}\right)$ is the (budget or transmission delay) balancing component.

To close this section, let us briefly refer to the related traffic engineering aspects. By Theorem 1 in the equilibrium, source $i$ transmits packets at rate $\bar{x}_{i}$ if it incurs the transmission cost $\bar{\eta}_{i}\left(\bar{\lambda}_{i}, \bar{x}_{i}\right)$. The corresponding charge per unit traffic flow for $i$ should therefore be defined by

$$
\lambda_{i}^{*}=\max \left\{\bar{\lambda}_{i}-\bar{h}_{i}\left(\bar{x}_{i}\right) / \bar{x}_{i}, 0\right\} .
$$

This result can be given the following interpretation. Suppose that $\lambda_{i}^{*}>0$ with $\bar{h}_{i}\left(\bar{x}_{i}\right)>0$ for some $\bar{x}_{i}>0$. Under these assumptions, source $i$ adjusts its rate to $\bar{x}_{i}$ if it observes the average delay $\lambda_{i}^{*}<\bar{\lambda}_{i}$, which corresponds to $\theta_{i}^{*}=\bar{\theta}_{i}-\bar{h}_{i}\left(\bar{x}_{i}\right)<\bar{\theta}_{i}$ packets maintained in the buffers along the routing paths for $w_{i}^{*}=\bar{w}_{i}-\bar{h}_{i}\left(\bar{x}_{i}\right)<\bar{w}_{i}$. Hence, the network motivates the source to optimally adjust its rate by providing to it the Quality of Service (QoS) parameters that are improved in comparison to those arising as a solution to $\operatorname{SYSTEM}(\mathbf{U}, \mathbf{A}, \mathbf{c})$. This implies that the network must be capable of providing differentiated services to the interacting sources, for example, by applying suitable Active Queue Management (AQM) techniques. It should also be noticed that for the equilibrium to be practically implementable it is necessary that the total amount of buffering in the network be at least 
$\sum_{i=1}^{n} \theta_{i}^{*}$, i.e., the (budget) balancing condition must be satisfied. To our best knowledge, the above requirements can be supported by the currently available networking technology. However, all in all, it should be noticed that the traffic engineering cost imposed by the mechanism may be substantial.

\section{Summary}

The problem of Nash equilibrium design has been intensively studied for decades. It should be recognized that there are many well known results establishing the existence and uniqueness of such constructions (see, e.g., Hurwicz, 1979; Maskin, 1999; Rosen, 1965; Green and Laffont, 1979; Laffont and Martimort, 2002; Myerson, 1981). Results presented in this paper expand this collection by illustrating the relationship between the properties of Lagrange multipliers, the Nash equilibrium of the addressed price-anticipation game and the condition of incentive compatibility. In this respect they may be viewed as an attempt to generalize some recent contributions on the design of networking games with price-anticipating agents (Johari and Tsitsiklis, 2009; Johari, 2004; Yang and Hajek, 2007; 2005; Maheswaran and Basar, 2004).

In our studies we have also referred to the control-theoretic design of price-based coordination rules in hierarchical systems (Findeisen, 1968; Findeisen et al., 1978; 1980; Apcan et al., 2010). The incentive compatibility conditions, which we have derived for the class of price-anticipation games, were applied in a study of the corresponding mechanism design costs. These costs can be intuitively related to the information monopoly that exists in the examined class of systems. Namely, each agent is capable of taking advantage of privately held information that is required to solve the interaction balancing (coordination) problem. Since it is not possible to fully eliminate the costs of enforcing incentive compatibility, they may play the key role in the mechanism design decision-making process.

Examples that we have given show that it is possible to distribute them among the agents interacting in the system. However, imposing incentive compatibility constraints may be an option for the coordinator only if the balancing condition can be satisfied. On the other hand, the coordinator may sacrifice the incentive compatibility property and introduce balanced mechanisms that are characterized by acceptable implementation costs (or efficiency loss). For more recent results in this subject, see the works of Johari and Tsitsiklis (2004) as well as Johari et al. (2005).

Finally, we have discussed an example of the engineering application of the developed analytic framework. Namely, we have discussed the design of TCP congestion control algorithms to illustrate how price-anticipation games may be induced in hierarchical systems.

\section{Acknowledgment}

This research was supported by the Polish Ministry of Science and Higher Education under the grant no. N N514 044438 .

\section{References}

Alpcan, T. and Pavel, L. (2009). Nash equilibrium design and optimization, International Conference on Game Theory for Networks, GameNets' 09, Istanbul, Turkey, pp. 164-170.

Alpcan, T., Pavel, L. and Stefanovic, N. (2010). An optimization and control theoretic approach to noncooperative game design, arXiv:1007.0144.

Arrow, K.J. and Hurwicz, L. (1977). Studies in Resource Allocation Processes, Cambridge University Press, New York, NY.

Basar, T. and Olsder, G. J. (1999). Dynamic Noncooperative Game Theory, SIAM Classics in Applied Mathematics, SIAM, Philadelphia, PA.

Bertsekas, D. and Ozdaglar, A. (2002). Pseudonormality and a Lagrange multiplier theory for constrained optimization, Journal of Optimization Theory and Applications 114(2): 287-343.

Chao, H. and Guo, X. (2002). Quality of Service Control in High-Speed Networks, John Wiley \& Sons, New York, NY.

Clempner, J.B. and Poznyak, A.S. (2011). Convergence method, properties and computational complexity for Lyapunov games, International Journal of Applied Mathematics and Computer Science 21(2): 349-361, DOI: 10.2478/v10006-011-0026-x.

Cramton, P. (1997). The FCC spectrum auctions: An early assessment, Journal of Economics and Management Strategy 6(3): 431-495.

Fiacco, A.V. and McCormick, G.P. (1990). Nonlinear Programming: Sequential Unconstrained Minimization Techniques, SIAM Classics in Applied Mathematics, SIAM, Philadelphia, PA.

Findeisen, W. (1968). Parametric optimization by primal method in multilevel systems, IEEE Transactions on Systems Science and Cybernetics 4(2): 155-164.

Findeisen, W., Bailey, F.N., Brdyś, M., Malinowski, K., Tatjewski, P. and Woźniak, A. (1980). Control and Coordination in Hierarchical Systems, John Wiley \& Sons, New York, NY.

Findeisen, W., Brdyś, M., Malinowski, K., Tatjewski, P. and Woźniak, A. (1978). On-line hierarchical control for steady-state systems, IEEE Transactions on Automatic Control 23(2): 189-209.

Fudenberg, D. and Tirole, J. (1991). Game Theory, The MIT Press, Cambridge, MA. 
Green, J.R. and Laffont, J.-J. (1979). Incentives in Public Decision-making, North-Holland Publishing Company, Amsterdam.

Grossman, S.J. and Stiglitz, J. (1980). On the impossibility of informationally efficient markets, American Economic Review 70(3): 393-408.

Groves, T., Radner, R. and Reiter, S. (Eds.) (1987). Information, Incentives, and Economic Mechanisms: Essays in Honor of Leonid Hurwicz, University of Minnesota Press, Mineapolis, MN.

Hurwicz, L. (1977). On Informationally Decentralized Systems, Studies in Resource Allocation Processes, Cambridge University Press, New York, NY, Chapter 4, pp. 425-459.

Hurwicz, L. (1979). On allocations attainable through Nash equilibria, Journal of Economic Theory 21(1): 140-165.

Hurwicz, L., Maskin, E. and Postlewaite, A. (1995). Feasible implementation of social choice correspondences by Nash equilibria, in J.O. Ledyard (Ed.), Essays in Honor of Stanley Reiter, Kluwer Academic Publishers, Norwell, MA, pp. 367-433.

Hurwicz, L. and Walker, M. (1990). On the generic nonoptimality of dominant-strategy allocation mechanisms: A general theorem that includes pure exchange economies, Econometrica 58(3): 683-704.

Jin, C., Wei, D., Low, S., Bunn, J., Choe, H., Doylle, J., Newman, H., Ravot, S., Singh, S. and Paganini, F. (2005). FAST TCP: From theory to experiments, IEEE Network 19(1): 4-11.

Jofré, A., Rockafellar, R. and Wets, R. (2007). Variational inequalities and economic equilibrium, Mathematics of Operations Research 32(1): 32.

Johari, R. (2004). Efficiency Loss in Market Mechanisms for Resource Allocation, Ph.D. thesis, MIT, Cambridge, MA.

Johari, R., Mannor, S. and Tsitsiklis, J.N. (2005). Efficiency loss in a network resource allocation game: The case of elastic supply, IEEE Transactions on Automatic Control 50(11): 1712-1724.

Johari, R. and Tsitsiklis, J.N. (2004). Efficiency loss in a network resource allocation game, Mathematics of Operation Research 29(3): 407-435.

Johari, R. and Tsitsiklis, J.N. (2009). Efficiency of scalar-parameterized mechanisms, Operations Research 57(4): 823-839.

Kakutani, S. (1941). A generalization of Brouwer's fixed point theorem, Duke Mathematical Journal 8(3): 457-459.

Karpowicz, M. (2010). Coordination in Hierarchical Systems with Rational Agents, Ph.D. thesis, Warsaw University of Technology, Warsaw.

Karpowicz, M. (2011). Designing auctions: A historical perspective, Journal of Telecommunications and Information Technology 3: 114-122.

Kelly, F.P. (1997). Charging and rate control for elastic traffic, European Transactions on Telecommunications 8(1): 33-37.
Kelly, F.P., Maulloo, A.K. and Tan, D.K. (1998). Rate control for communication networks: Shadow prices, proportional fairness, and stability, Journal of the Operational Research Society 49(3): 237-252.

Kołodziej, J. and Xhafa, F. (2011). Modern approaches to modeling user requirements on resource and task allocation in hierarchical computational grids, International Journal of Applied Mathematics and Computer Science 21(2): 243-257, DOI: 10.2478/v10006-011-0018-x.

Krishna, V. (2002). Auction Theory, Academic Press, San Diego, CA.

La, R.J. and Anantharam, V. (2000). Charge-sensitive TCP and rate control in the Internet, IEEE INFOCOM 2000, TelAviv, Israel, pp. 1166-1175.

Laffont, J.-J. and Martimort, D. (2002). The Theory of Incentives, Princeton University Press, Princeton, NJ.

Low, S.H. (2003). A duality model of TCP and queue management algorithms, IEEE/ACM Transactions on Networking 11(4): 525-536.

Low, S.H. and Lapsley, D.E. (1999). Optimization flow control, I: Basic algorithm and convergence, IEEE/ACM Transactions on Networking 7(6): 861-874.

Low, S., Paganini, F., Wang, J. and Doyle, J. (2003). Linear stability of TCP/RED and a scalable control, Computer Networks 43(5): 633-647.

Low, S., Peterson, L. and Wang, L. (2002). Understanding TCP Vegas: A duality model, Journal of the ACM (JACM) 49(2): 207-235.

Lubacz, J. (Ed.) (2011). Auction Mechanisms in Telecommunications, WKŁ, Warsaw, (in Polish).

Maheswaran, R. and Basar, T. (2004). Social welfare of selfish agents: Motivating efficiency for divisible resources, Proceedings of the 43rd IEEE Conference on Decision and Control, The Bahamas.

Malinowski, K. (2002). Optimization network flow control and price coordination with feedback: Proposal of a new distributed algorithm, Computer Communications 25(11-12): 1028-1036

Mas-Colell, A., Whinston, M.D. and Green, J.R. (1995). Microeconomic Theory, Oxford University Press, New York, NY.

Maskin, E. (1999). Nash equilibrium and welfare optimality, The Review of Economic Studies 66(1): 23-38.

Milgrom, P. (2004). Putting Auction Theory to Work, Cambridge University Press, New York, NY.

Milgrom, P. and Weber, R. (1982). A theory of auctions and competitive bidding, Econometrica 50(5): 1089-1122.

Mo, J. and Walrand, J. (2000). Fair end-to-end window-based congestion control, IEEE/ACM Transactions on Networking 8(5): 556-567.

Myerson, R.B. (1981). Optimal auction design, Mathematics of Operations Research 6(1): 58-73.

Myerson, R.B. (1991). Game Theory: Analysis of Conflict, Harvard University Press, Cambridge, MA. 
Nash, J. (1950). Equilibrium points in n-person games, Proceedings of National Academy of Science 36(1): 48-49.

Nash, J. (1951). Non-cooperative games, Annals of Mathematics 54(2): 289-295.

Negishi, T. (1960). Welfare economics and existence of an equilibrium for a competitive economy, Metroeconomica 12(2-3): 92-97.

Ogryczak, W., Pióro, M. and Tomaszewski, A. (2005). Telecommunications network design and max-min optimization problem, Journal of Telecommunications and Information Technology 3: 43-56.

Ogryczak, W., Wierzbicki, A. and Milewski, M. (2008). A multi-criteria approach to fair and efficient bandwidth allocation, Omega 36(3): 451-463.

Pióro, M. and Medhi, D. (2004). Routing, Flow, and Capacity Design in Communication and Computer Networks, Morgan Kaufmann, San Francisco, CA.

Rockafellar, R.T. and Wets, R.J.-B. (2004). Variational Analysis, A Series of Comprehensive Studies in Mathematics, Vol. 317, Springer-Verlag, Berlin/Heidelberg.

Rosen, J.B. (1965). Existence and uniqueness of equilibrium points for concave n-person games, Econometrica 33(3): 520-534.

Rotschild, M. and Stiglitz, J. (1976). Equilibrium in competitive insurance markets: An essay on the economics of imperfect information, Quarterly Journal of Economics 90(4): 630-649.

Sen, A. (1969). Quasi-transitivity, rational choice and collective decisions, Review of Economic Studies 36(107): 381-93.

Sen, A. (1970). Interpersonal aggregation and partial comparability, Econometrica 38(3): 393-409.

Sen, A. (1977). On weights and measures: Informational constraints in social welfare, Econometrica 45(7): 1539-1572.

Srikant, R. (2003). The Mathematics of Internet Congestion Control, Birkhäuser, Boston, MA.

Stallings, W. (1998). High-Speed Networks, Prentice Hall, Upper Saddle River, NJ.

Stiglitz, J. (2000). The contributions of the economics of information to twentieth century economics, The Quarterly Journal of Economics 115(4): 1441-1478.
Uzawa, H. (1960). Market mechanisms and mathematical programming, Econometrica 28(4): 872-881.

Vickrey, W. (1961). Counterspeculation, auctions and competitive sealed tenders, Journal of Finance 16(1): 8-37.

Wei, D.X., Jin, C., Low, S.H. and Hegde, S. (2006). FAST TCP: Motivation, architecture, algorithms, performance, IEEE/ACM Transactions on Networking 16(6): 1246-1259.

Wierzbicki, A. P., Makowski, M. and Wessels, J. (2001). ModelBased Decision Support Methodology with Environmental Applications, Kluwer Academic Publishers, Dordrecht.

Yang, S. and Hajek, B. (2005). Revenue and stability of a mechanism for efficient allocation of a divisible good, Mimeo, University of Illinois, Urbana-Champaign, IL.

Yang, S. and Hajek, B. (2007). VCG-Kelly mechanisms for allocation of divisible goods: Adapting VCG mechanisms to one-dimensional signals, IEEE Journal on Selected Areas in Communications 25(6): 1237-1243.

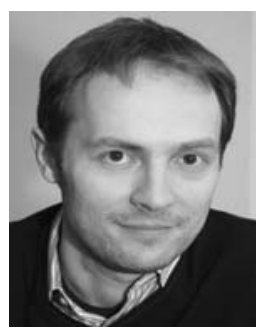

Michał P. Karpowicz received a Ph.D. in computer science from the Warsaw University of Technology, Poland, in 2010. Currently he is an assistant professor at the Research and Academic Computer Network (NASK). His research interests focus on game theory, network control and optimization.
Received: 15 November 2011

Revised: 10 May 2012

Re-revised: 11 June 2012 